\title{
Synthesis of Some Aroylhydrazones and 2,5-Disubstituted-1,3,4- Oxadiazoles as DNA Photocleaving Agents
}

Vinod Kumar $^{1 *}$, Mohit Kumar ${ }^{1}$, Vikas Beniwal ${ }^{2}$, Girish Kumar Gupta ${ }^{3}$, Sunil Kumar ${ }^{4}$ and Ramesh Kataria ${ }^{5}$

${ }^{1}$ Department of Chemistry, Maharishi Markandeshwar University, Mullana, Ambala, Haryana, India

${ }^{2}$ Department of Biotechnology, Maharishi Markandeshwar University, Mullana, Ambala, Haryana, India

${ }^{3}$ Department of Pharmaceutical Chemistry, Maharishi Markandeshwar College of Pharmacy, Maharishi Markandeshwar University, Mullana, Ambala, Haryana, India

${ }^{4}$ Department of Chemistry, Government Girls PG College, Hisar, Haryana, India

${ }^{5}$ Department of Chemistry, Panjab University, Chandigarh, India

\begin{abstract}
Some 2,5-disubstituted-1,3,4-oxadiazole derivatives have been synthesized conveniently via oxidative cyclization of various synthesized aroylhydrazones by (diacetoxyiodo)benzene in dichloromethane under mild reaction conditions. In addition, the effect of electron-withdrawing/releasing groups on the formation of oxadiazole nucleus has also been studied. Compounds were obtained in good yields and their structures have been established on the basis of their FT-IR, ${ }^{1} \mathrm{H},{ }^{13} \mathrm{C}$ NMR and mass spectral data. Herein, a total of 42 compounds (hydrazones as well as oxadiazoles) were synthesized and investigated for their DNA photocleavage potential using plasmid DNA. It has been observed that aroylhydrazones showed good DNA photocleavage activity in comparison to their corresponding oxadiazoles.
\end{abstract}

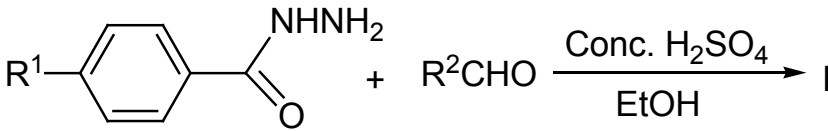<smiles>[R]C(C)=NNC(=O)c1ccc([R])cc1</smiles><smiles></smiles>

Keywords: Oxadiazole; Pyrazole; Aroylhydrazone; (Diacetoxyiodo) benzene; DNA photocleavage

\section{Introduction}

Azoles are well known for their huge contribution in the pharmaceutical sector and among them pyrazole is still being considered as one of the leading pharmacophores in the development of bioactive compounds [1-10]. Pyrazole derivatives exhibited a broad spectrum of useful properties such as antitumor [11], antitubercular [12], antioxidant [13], anti-inflammatory [14,15], antibacterial [16], anti-obesity [17] and antidepressant [18] activities. Similarly, 1,3,4-oxadiazoles have been reported to exhibit various biological $[19,20]$ and pharmacological activities [21-25]. These compounds, in particular, have been found to exhibit biological potential such as anticancer activity [26-28] specifically in the presence of some potent heterocyclic nuclei. In the past decade much attention has been paid towards the evaluation of the DNA photo cleavage potential of a variety of oxadiazole derivatives [29-31]. DNA is a primary site where most of the drugs interact and lead to inhibition or death of cancerous cells [32]. Therefore, compounds having binding or interacting ability with the DNA structure could be used as probes for DNA structure, as potential chemotherapeutic and diagnostic agents [33]. In view of the above facts, it was decided to synthesize some novel 2,5-disubstituted- 1,3,4-oxadiazoles under mild reaction conditions and evaluate their DNA photo cleavage activity.

\section{Experimental}

Melting points of all the synthesized compounds were determined in an open capillary using digital melting point apparatus and are uncorrected. IR spectra were recorded as $\mathrm{KBr}$ discs on a Perkin-Elmer Spectrophotometer in the 4,000-450 $\mathrm{cm}^{-1}$ range. Both ${ }^{1} \mathrm{H}$ and ${ }^{13} \mathrm{C}$ NMR spectra of the compounds were recorded on a Bruker Advance NMR Spectrophotometer at $400 \mathrm{MHz}$ and $100 \mathrm{MHz}$, respectively. Chemical shifts were measured relative to internal reference standard,

*Corresponding author: Vinod Kumar, Department of Chemistry, Maharish Markandeshwar University, Mullana, Ambala-133 207, Haryana, India, Tel: +918059444494; E-mail: vinodbatan@gmail.com

Received June 13, 2016; Accepted July 04, 2016; Published July 08, 2016

Citation: Kumar V, Kumar M, Beniwal V, Gupta GK, Kumar S, et al. (2016) Synthesis of Some Aroylhydrazones and 2,5-Disubstituted-1,3,4-Oxadiazoles as DNA Photocleaving Agents. Med chem (Los Angeles) 6: 474-485. doi:10.4172/21610444.1000386

Copyright: $\odot 2016$ Kumar V, et al. This is an open-access article distributed under the terms of the Creative Commons Attribution License, which permits unrestricted use, distribution, and reproduction in any medium, provided the original author and source are credited. 
Citation: Kumar V, Kumar M, Beniwal V, Gupta GK, Kumar S, et al. (2016) Synthesis of Some Aroylhydrazones and 2,5-Disubstituted-1,3,4Oxadiazoles as DNA Photocleaving Agents. Med chem (Los Angeles) 6: 474-485. doi:10.4172/2161-0444.1000386

tetramethylsilane (TMS) $(\delta=0)$ in $\mathrm{CDCl}_{3}$ or DMSO- $d_{6}$, and were reported on $\delta$ scale (ppm). Coupling constants $(J)$ were given in $\mathrm{Hz}$. Mass spectra were recorded on Waters, Q-Tof micromass, ESI source, Mass Spectrometer. Carbon, nitrogen, hydrogen contents were analyzed using LECO 9320 analyzer. Aroylhydrazines 1 [34], 4-formylpyrazoles 2 [35] utilized in present investigation were synthesized according to the literature methods.

\section{Synthesis of Aroylhydrazones (3a-u)}

\section{General procedure}

A solution of 4 -formylpyrazole or substituted benzaldehyde $(0.01 \mathrm{~mol})$ and sulfuric acid (one drop) in dichloromethane (DCM) was added to an ethanolic solution of aroylhydrazine $(1,0.01 \mathrm{~mol})$ under stirring. Then reaction mass was refluxed for $40-45 \mathrm{~min}$ till completion of the reaction. The reaction was monitored by thin layer chromatography, excess of solvent was distilled out, and then the reaction mass was cooled to room temperature. The obtained product was filtered, washed with alcohol and recrystallised from ethanol. The melting points were noted before submitting the samples for analysis.

\section{N-Benzoyl-N'-(1',3'-diphenyl-4'-pyrazolylmethylidene)} hydrazine (3a)

Yield 89 \%; m.p.: (Obs.) $180-181^{\circ} \mathrm{C}$, m.p.: (Lit.) $180-182{ }^{\circ} \mathrm{C}$; TLC $\mathrm{R}_{\mathrm{f}}=0.37$ [ethylacetate: hexane (3:7)]; ${ }^{1} \mathrm{H}-\mathrm{NMR}\left(400 \mathrm{MHz}, \mathrm{DMSO}-d_{6}\right)$ : $\delta=11.76\left(\mathrm{~s}, 1 \mathrm{H}, \mathrm{H}-\mathrm{N}, \mathrm{D}_{2} \mathrm{O}\right.$ exchangeable), $8.96\left(\mathrm{~s}, 1 \mathrm{H}, \mathrm{H}-5^{\prime}\right), 8.61(\mathrm{~s}$, 1H, H-6'), 7.92-8.00 (m, 4H, H-2, H-6 \& H-2", H-6"), 7.74 (d, 2H, J = 7.1 Hz, H-2"', H-6"'), 7.35-7.60 (m, 9H, H-3, H-4, H-5, H-Ph" \& $\mathrm{H}-\mathrm{Ph}$ "') ppm; ${ }^{13} \mathrm{C}-\mathrm{NMR}\left(100 \mathrm{MHz}, \mathrm{CDCl}_{3}+\mathrm{DMSO}_{-} d_{6}\right): \delta=162.9(\mathrm{C}$, C-7), 151.8 (C, C-3'), 141.0 (CH, C-6'), 139.0 (C, C-1"), 133.3 (C, C-1), 132.0 (CH, C-4), 131.5 (C, C-1"'), 129.4 (CH, C-3"', C-5"'), $128.6(\mathrm{CH}$, C-2, C-6), 128.4 (CH, C-3", C-5"), 128.3 (CH, C-4"'), 128.2 (CH, C-2"', C-6"'), 127.6 (CH, C-3, C-5), 127.5 (CH, C-5'), 126.8 (CH, C-4"), 118.7 (CH, C-2", C-6"), 116.9 (C, C-4') ppm; FT-IR (KBr): 3427 (N-H str.), 1668 (C=O str.) $\mathrm{cm}^{-1}$; MS (ESI): $\mathrm{m} / \mathrm{z}=367.2(\mathrm{M}+1)^{+}$; Anal. Calcd. for $\mathrm{C}_{23} \mathrm{H}_{18} \mathrm{~N}_{4} \mathrm{O}: \mathrm{C}, 75.37 ; \mathrm{H}, 4.91 ; \mathrm{N}, 15.29$. Found: $\mathrm{C}, 75.34 ; \mathrm{H}, 4.89 ; \mathrm{N}$, 15.27 .

\section{N-Benzoyl-N'-(4'-nitrobenzylidene)hydrazine (3b)}

Yield $86.8 \%$; m.p.: $238-239^{\circ} \mathrm{C}$; TLC $\mathrm{R}_{\mathrm{f}}=0.39$ [ethylacetate: hexane (3:7)]; ${ }^{1} \mathrm{H}-\mathrm{NMR}\left(400 \mathrm{MHz}, \mathrm{DMSO}-d_{6}\right): \delta=11.78\left(\mathrm{~s}, 1 \mathrm{H}, \mathrm{H}-\mathrm{N}, \mathrm{D}_{2} \mathrm{O}\right.$ exchangeable), 8.61 (s, $\left.1 \mathrm{H}, \mathrm{H}-7^{\prime}\right), 8.34-8.48$ (m, $\left.4 \mathrm{H}, \mathrm{H}-\mathrm{Ph}^{\prime}\right), 7.25-$ $7.78(\mathrm{~m}, 5 \mathrm{H}, \mathrm{H}-\mathrm{Ph}) \mathrm{ppm} ;{ }^{13} \mathrm{C}-\mathrm{NMR}\left(100 \mathrm{MHz}, \mathrm{DMSO}-d_{6}\right): \delta=163.0$ (C, C-7), 148.0 (C, C-4'), 141.5 (C, C-1'), 140.8 (CH, C-7'), 132.8 (C, C-1), 132.4 (CH, C-4), 128.5 (CH, C-2, C-6), 127.7 (CH, C-2', C-6'), 127.4 (CH, C-3, C-5), 124.1 (CH, C-3', C-5') ppm; FT-IR (KBr): 3430 (N-H str.), 1668 (C=O str.), $1540\left(\mathrm{NO}_{2}\right.$ asymmetric str.), $1351\left(\mathrm{NO}_{2}\right.$ symmetric str.) $\mathrm{cm}^{-1}$; MS (ESI): $\mathrm{m} / \mathrm{z}=270.3(\mathrm{M}+1)^{+}$; Anal. Calcd. for $\mathrm{C}_{14} \mathrm{H}_{11} \mathrm{~N}_{3} \mathrm{O}_{3}: \mathrm{C}, 62.38 ; \mathrm{H}, 4.08 ; \mathrm{N}, 15.59$. Found: $\mathrm{C}, 62.36 ; \mathrm{H}, 4.08 ; \mathrm{N}$, 15.58 .

\section{N-Benzoyl-N'-(4'-bromobenzylidene)hydrazine (3c)}

Yield $87.5 \%$; m.p.: $201-202{ }^{\circ} \mathrm{C}$; TLC $\mathrm{R}_{\mathrm{f}}=0.51$ [ethylacetate: hexane (3:7)]; ${ }^{1} \mathrm{H}-\mathrm{NMR}\left(400 \mathrm{MHz}, \mathrm{DMSO}-d_{6}\right): \delta=11.80\left(\mathrm{~s}, 1 \mathrm{H}, \mathrm{H}-\mathrm{N}, \mathrm{D}_{2} \mathrm{O}\right.$ exchangeable), 8.48 (s, 1H, H-7'), 7.89 (d, 2H, $J=7.4 \mathrm{~Hz}, \mathrm{H}-2, \mathrm{H}-6$ ), 7.31-7.75 (m, 7H, H-Ph' \& H-3, H-4, H-5) ppm; ${ }^{13} \mathrm{C}-\mathrm{NMR}(100 \mathrm{MHz}$, DMSO- $\left.d_{6}\right): \delta=162.9$ (C, C-7), $141.2\left(\mathrm{CH}, \mathrm{C}-7^{\prime}\right), 133.6\left(\mathrm{CH}, \mathrm{C}-3^{\prime}, \mathrm{C}-5^{\prime}\right)$, 133.1 (C, C-1), 132.2 (CH, C-4), 130.1 (C, C-1'), 128.4 (CH, C-2, C-6), 127.8 (CH, C-3, C-5), 127.4 (CH, C-2', C-6'), 123.1 (C, C-4') ppm; FTIR (KBr): 3433 (N-H str.), 1665 (C=O str.) cm ${ }^{-1}$; MS (ESI): $\mathrm{m} / \mathrm{z}=303.4$ $(\mathrm{M}+1)^{+}, 305.5(\mathrm{M}+2)^{+}$in the ratio showing typical bromine isotope profile (1:1); Anal. Calcd. for $\mathrm{C}_{14} \mathrm{H}_{11} \mathrm{BrN}_{2} \mathrm{O}: \mathrm{C}, 55.55 ; \mathrm{H}, 3.64 ; \mathrm{N}, 9.26$. Found: C, 55.54; H, 3.62; N, 9.23.

\section{N-Benzoyl-N'-(4'-methoxybenzylidene)hydrazine (3d)}

Yield $87.9 \%$; m.p.: $189-190^{\circ} \mathrm{C}$; TLC $\mathrm{R}_{\mathrm{f}}=0.35$ [ethylacetate: hexane (3:7)]; ${ }^{1} \mathrm{H}-\mathrm{NMR}\left(400 \mathrm{MHz}, \mathrm{DMSO}-d_{6}\right): \delta=11.94\left(\mathrm{~s}, 1 \mathrm{H}, \mathrm{H}-\mathrm{N}, \mathrm{D}_{2} \mathrm{O}\right.$ exchangeable), 8.42 (s, 1H, H-7'), 7.32-7.92 (m, 7H, H-2', H-6' \& H-Ph), $6.99\left(\mathrm{~d}, 2 \mathrm{H}, J=8.6 \mathrm{~Hz}, \mathrm{H}-3^{\prime}, \mathrm{H}-5^{\prime}\right), 3.79\left(\mathrm{~s}, 3 \mathrm{H}, 4^{\prime}-\mathrm{OCH}_{3}\right) \mathrm{ppm} ;{ }^{13} \mathrm{C}-\mathrm{NMR}$ (100 MHz, DMSO- $\left.d_{6}\right): \delta=161.4$ (C, C-7), 160.0 (C, C-4'), $140.8(\mathrm{CH}$, C-7'), 132.9 (C, C-1), 132.0 (CH, C-4), 129.1 (CH, C-2', C-6'), 128.1 (CH, C-2, C-6), 127.8 (CH, C-3, C-5), 122.4 (C, C-1'), 114.1 (CH, C-3', C-5'), $55.1\left(\mathrm{OCH}_{3}\right)$ ppm; FT-IR (KBr): 3421 (N-H str.), 1666 (C=O str.) $\mathrm{cm}^{-1}$; MS (ESI): $\mathrm{m} / \mathrm{z}=255.2(\mathrm{M}+1)^{+}$; Anal. Calcd. for $\mathrm{C}_{15} \mathrm{H}_{14} \mathrm{~N}_{2} \mathrm{O}_{2}: \mathrm{C}$, $70.81 ; \mathrm{H}, 5.51 ; \mathrm{N}, 11.01$. Found: C, 70.78; H, 5.50; N, 11.00 .

\section{N-Benzoyl-N'-(4'-methylbenzylidene)hydrazine (3e)}

Yield $88.7 \%$; m.p.: $195-196^{\circ} \mathrm{C}$; TLC $\mathrm{R}_{\mathrm{f}}=0.49$ [ethylacetate: hexane (3:7)]; ${ }^{1} \mathrm{H}-\mathrm{NMR}\left(400 \mathrm{MHz}, \mathrm{DMSO}-d_{6}\right): \delta=11.97\left(\mathrm{~s}, 1 \mathrm{H}, \mathrm{H}-\mathrm{N}, \mathrm{D}_{2} \mathrm{O}\right.$ exchangeable), 8.44 (s, 1H, H-7'), 7.40-7.93 (m, 7H, H-2', H-6' \& H-Ph), $7.23\left(\mathrm{~d}, 2 \mathrm{H}, J=8.4 \mathrm{~Hz}, \mathrm{H}-3{ }^{\prime}, \mathrm{H}-5^{\prime}\right), 2.34$ (s, 3H, 4'-CH $)$ ppm; ${ }^{13} \mathrm{C}-\mathrm{NMR}$ $\left(100 \mathrm{MHz}, \mathrm{DMSO}-d_{6}\right): \delta=161.0(\mathrm{C}, \mathrm{C}-7), 140.7\left(\mathrm{CH}, \mathrm{C}-7^{\prime}\right), 140.1(\mathrm{C}$, C-4'), 132.2 (C, C-1), 131.9 (CH, C-4), 129.4 (CH, C-2', C-6'), 129.2 (CH, C-3', C-5'), 128.6 (CH, C-2, C-6), 128.5 (C, C-1'), 127.5 (CH, C-3, C-5), $21.1\left(\mathrm{CH}_{3}\right)$ ppm; FT-IR (KBr): 3425 (N-H str.), 1665 (C=O str.) $\mathrm{cm}^{-1}$; MS (ESI): $\mathrm{m} / \mathrm{z}=239.2(\mathrm{M}+1)^{+}$; Anal. Calcd. for $\mathrm{C}_{15} \mathrm{H}_{14} \mathrm{~N}_{2} \mathrm{O}: \mathrm{C}$, 75.57; H, 5.88; N, 11.75. Found: C, 75.54; H, 5.87; N, 11.72.

\section{N-Benzoyl-N'-(thien-2'-yl-methylidene)hydrazine (3f)}

Yield $86.5 \%$; m.p.: $149-150^{\circ} \mathrm{C}$; TLC $\mathrm{R}_{\mathrm{f}}=0.36$ [ethylacetate: hexane (3:7)]; ${ }^{1} \mathrm{H}-\mathrm{NMR}\left(400 \mathrm{MHz}, \mathrm{DMSO}-d_{6}\right): \delta=11.82\left(\mathrm{~s}, 1 \mathrm{H}, \mathrm{H}-\mathrm{N}, \mathrm{D}_{2} \mathrm{O}\right.$ exchangeable), 8.69 (s, $\left.1 \mathrm{H}, \mathrm{H}-6^{\prime}\right), 7.91(\mathrm{~d}, 2 \mathrm{H}, J=7.3 \mathrm{~Hz}, \mathrm{H}-2, \mathrm{H}-6)$, 7.11-7.61 (m, 6H, H-3, H-4, H-5 \& H-3', H-4', H-5') ppm; ${ }^{13} \mathrm{C}-\mathrm{NMR}$ $\left(100 \mathrm{MHz}, \mathrm{DMSO}-d_{6}\right): \delta=163.1(\mathrm{C}, \mathrm{C}-7), 142.9\left(\mathrm{CH}, \mathrm{C}-6^{\prime}\right), 139.1(\mathrm{CH}$, C-5'), 133.3 (C, C-1), 131.6 (CH, C-4), 130.6 (C, C-2'), 128.6 (CH, C-4'), $128.3(\mathrm{CH}, \mathrm{C}-2, \mathrm{C}-6), 127.6(\mathrm{CH}, \mathrm{C}-3$ '), $127.5(\mathrm{CH}, \mathrm{C}-3, \mathrm{C}-5) \mathrm{ppm}$; FT-IR (KBr): 3421 (N-H str.), 1665 (C=O str.) cm ${ }^{-1}$; MS (ESI): $\mathrm{m} / \mathrm{z}=$ $231.1(\mathrm{M}+1)^{+}$; Anal. Calcd. for $\mathrm{C}_{12} \mathrm{H}_{10} \mathrm{~N}_{2} \mathrm{OS}: \mathrm{C}, 62.58 ; \mathrm{H}, 4.34 ; \mathrm{N}, 12.17$. Found: C, 62.57; H, 4.33; N, 12.15 .

\section{N-Benzoyl-N'-(furl-2'-yl-methylidene)hydrazine (3g)}

Yield 87 \%; m.p.: $139-140{ }^{\circ} \mathrm{C}$; TLC $\mathrm{R}_{\mathrm{f}}=0.32$ [ethylacetate: hexane (3:7)]; ${ }^{1} \mathrm{H}-\mathrm{NMR}\left(400 \mathrm{MHz}, \mathrm{DMSO}-d_{6}\right): \delta=11.85\left(\mathrm{~s}, 1 \mathrm{H}, \mathrm{H}-\mathrm{N}, \mathrm{D}_{2} \mathrm{O}\right.$ exchangeable), 8.72 (s, $\left.1 \mathrm{H}, \mathrm{H}-6^{\prime}\right), 7.89(\mathrm{~d}, 2 \mathrm{H}, J=7.4 \mathrm{~Hz}, \mathrm{H}-2, \mathrm{H}-6)$, 7.09-7.58 (m, 6H, H-3, H-4, H-5 \& H-3', H-4', H-5') ppm; ${ }^{13} \mathrm{C}-\mathrm{NMR}$ $\left(100 \mathrm{MHz}, \mathrm{DMSO}-d_{6}\right): \delta=162.5(\mathrm{C}, \mathrm{C}-7), 144.1(\mathrm{CH}, \mathrm{C}-6)$ ), $139.3(\mathrm{C}$, C-2'), $138.8\left(\mathrm{CH}, \mathrm{C}^{\prime} 5^{\prime}\right), 132.6(\mathrm{C}, \mathrm{C}-1), 131.8(\mathrm{CH}, \mathrm{C}-4), 127.9(\mathrm{CH}$ C-2, C-6), 126.8 (CH, C-3, C-5), $113.9\left(\mathrm{CH}, \mathrm{C}-4\right.$ '), $112.3\left(\mathrm{CH}, \mathrm{C}-3^{\prime}\right)$ ppm; FT-IR (KBr): 3422 (N-H str.), 1663 (C=O str.) $\mathrm{cm}^{-1}$; MS (ESI): $\mathrm{m} / \mathrm{z}=215.3(\mathrm{M}+1)^{+}$; Anal. Calcd. for $\mathrm{C}_{12} \mathrm{H}_{10} \mathrm{~N}_{2} \mathrm{O}_{2}: \mathrm{C}, 67.19 ; \mathrm{H}, 4.67 ; \mathrm{N}$, 13.06. Found: C, $67.18 ; \mathrm{H}, 4.65 ; \mathrm{N}, 13.05$.

\section{$\mathrm{N}^{\prime}$-( 1',3'-Diphenyl-4'-pyrazolylmethylidene)-N-(4- methylbenzoyl)hydrazine ( $3 \mathrm{~h}$ )}

Yield 88 \%; m.p.: $192-193{ }^{\circ} \mathrm{C}$; TLC $\mathrm{R}_{\mathrm{f}}=0.30$ [ethylacetate: hexane (3:7)]; ${ }^{1} \mathrm{H}-\mathrm{NMR}\left(400 \mathrm{MHz}, \mathrm{DMSO}-d_{6}\right): \delta=11.70\left(\mathrm{~s}, 1 \mathrm{H}, \mathrm{H}-\mathrm{N}, \mathrm{D}_{2} \mathrm{O}\right.$ exchangeable), $8.92\left(\mathrm{~s}, 1 \mathrm{H}, \mathrm{H}-5^{\prime}\right), 8.62\left(\mathrm{~s}, 1 \mathrm{H}, \mathrm{H}-6^{\prime}\right), 7.99(\mathrm{~d}, 2 \mathrm{H}, J=8.2$ $\mathrm{Hz}, \mathrm{H}-2$ ", H-6"), 7.85 (d, 2H, J = 8.0 Hz, H-2, H-6), 7.74 (d, 2H, J = 7.1 Hz, H-2"', H-6"'), 7.28-7.54 (m, 8H, H-3, H-5, H-Ph" \& H-Ph"'), 2.38 (s, $\left.3 \mathrm{H}, 4-\mathrm{CH}_{3}\right)$ ppm; ${ }^{13} \mathrm{C}-\mathrm{NMR}\left(100 \mathrm{MHz}, \mathrm{DMSO}-d_{6}\right): \delta=162.8(\mathrm{C}$, C-7), 151.8 (C, C-3'), 141.4 (CH, C-6'), 140.7 (C, C-4), 139.0 (C, C-1"), 
Citation: Kumar V, Kumar M, Beniwal V, Gupta GK, Kumar S, et al. (2016) Synthesis of Some Aroylhydrazones and 2,5-Disubstituted-1,3,4Oxadiazoles as DNA Photocleaving Agents. Med chem (Los Angeles) 6: 474-485. doi:10.4172/2161-0444.1000386

132.1 (C, C-1"'), 130.5 (C, C-1), 129.3 (CH, C-3"', C-5"'), $128.7(\mathrm{CH}$, C-2, C-6), 128.4 (CH, C-3", C-5"), 128.3 (CH, C-4"'), 128.2 (CH, C-2"', C-6"'), 127.4 (CH, C-3, C-5), 126.6 (CH, C-5'), 126.4 (CH, C-4"), 118.6 (CH, C-2", C-6"), 116.9 (C, C-4'), $21.2\left(\mathrm{CH}_{3}\right)$ ppm; FT-IR (KBr): 3415 (N-H str.), $1664\left(\mathrm{C}=\mathrm{O}\right.$ str.) $\mathrm{cm}^{-1}$; MS (ESI): $\mathrm{m} / \mathrm{z}=381.2(\mathrm{M}+1)^{+}$; Anal. Calcd. for $\mathrm{C}_{24} \mathrm{H}_{20} \mathrm{~N}_{4} \mathrm{O}: \mathrm{C}, 75.75 ; \mathrm{H}, 5.26 ; \mathrm{N}, 14.73$. Found: $\mathrm{C}, 75.74 ; \mathrm{H}$, $5.23 ; \mathrm{N}, 14.71$.

\section{4-Methyl-N'-(4'-nitrobenzylidene)-N-benzoylhydrazine (3i)}

Yield $85.9 \%$; m.p.: $189-190^{\circ} \mathrm{C}$; TLC $\mathrm{R}_{\mathrm{f}}=0.33$ [ethylacetate: hexane (3:7)]; ${ }^{1} \mathrm{H}-\mathrm{NMR}\left(400 \mathrm{MHz}, \mathrm{DMSO}-d_{6}\right): \delta=12.05\left(\mathrm{~s}, 1 \mathrm{H}, \mathrm{H}-\mathrm{N}, \mathrm{D}_{2} \mathrm{O}\right.$ exchangeable), 8.56 (s, $\left.1 \mathrm{H}, \mathrm{H}-7^{\prime}\right), 8.27$ (d, $\left.2 \mathrm{H}, J=8.6 \mathrm{~Hz}, \mathrm{H}-3^{\prime}, \mathrm{H}^{-5}\right)$, 7.85-8.00 (m, 4H, H-2, H-6 \& H-2', H-6'), 7.32 (d, 2H, $J=7.8 \mathrm{~Hz}, \mathrm{H}-3$, $\mathrm{H}-5), 2.41$ (s, 3H, 4-CH $)$ ppm; ${ }^{13} \mathrm{C}-\mathrm{NMR}\left(100 \mathrm{MHz}\right.$, DMSO- $\left.d_{6}\right): \delta=$ 163.3 (C, C-7), 147.7 (C, C-4'), 144.8 (C, C-4), 142.0 (C, C-1'), 140.7 (CH, C-7'), 130.1 (C, C-1), 128.8 (CH, C-2, C-6), 127.8 (CH, C-3, C-5), $127.7\left(\mathrm{CH}, \mathrm{C}-2^{\prime}, \mathrm{C}-6^{\prime}\right), 123.8\left(\mathrm{CH}, \mathrm{C}-3^{\prime}, \mathrm{C}-5^{\prime}\right), 21.1\left(\mathrm{CH}_{3}\right)$ ppm; FT-IR (KBr): 3433 (N-H str.), 1667 (C=O str.), 1543 ( $\mathrm{NO}_{2}$ asymmetric str.), $1349\left(\mathrm{NO}_{2}\right.$ symmetric str.) $\mathrm{cm}^{-1}$; MS (ESI): $\mathrm{m} / \mathrm{z}=284.3(\mathrm{M}+1)^{+}$; Anal. Calcd. for $\mathrm{C}_{15} \mathrm{H}_{13} \mathrm{~N}_{3} \mathrm{O}_{3}: \mathrm{C}, 63.54 ; \mathrm{H}, 4.59 ; \mathrm{N}, 14.82$. Found: $\mathrm{C}, 63.51 ; \mathrm{H}$, $4.58 ; \mathrm{N}, 14.82$.

\section{N'-(4'-Bromobenzylidene)-N-(4-methylbenzoyl)hydrazine (3j)}

Yield $89 \%$; m.p.: $231-232{ }^{\circ} \mathrm{C}$; TLC $\mathrm{R}_{\mathrm{f}}=0.39$ [ethylacetate: hexane (3:7)]; ${ }^{1} \mathrm{H}-\mathrm{NMR}\left(400 \mathrm{MHz}, \mathrm{DMSO}-d_{6}\right): \delta=11.79\left(\mathrm{~s}, 1 \mathrm{H}, \mathrm{H}-\mathrm{N}, \mathrm{D}_{2} \mathrm{O}\right.$ exchangeable), 8.41 (s, 1H, H-7'), 7.55-7.83 (m, 6H, H-2, H-6 \& H-Ph'), 7.27 (d, $2 \mathrm{H}, J=7.8 \mathrm{~Hz}, \mathrm{H}-3, \mathrm{H}-5), 2.38$ (s, 3H, 4-CH $)$ ppm; ${ }^{13} \mathrm{C}-\mathrm{NMR}$ $\left(100 \mathrm{MHz}, \mathrm{DMSO}-d_{6}\right): \delta=163.2(\mathrm{C}, \mathrm{C}-7), 146.2(\mathrm{C}, \mathrm{C}-4), 141.5(\mathrm{CH}$, C-7'), 133.5 (CH, C-3', C-5'), 131.5 (CH, C-2, C-6), 130.3 (C, C-1'), 129.9 (C, C-1), 128.7 (CH, C-3, C-5), 127.6 (CH, C-2', C-6'), 123.2 (C, C-4'), $21.1\left(\mathrm{CH}_{3}\right)$ ppm; FT-IR (KBr): 3436 (N-H str.), 1665 (C=O str.) $\mathrm{cm}^{-1}$; MS (ESI): $\mathrm{m} / \mathrm{z}=317.2(\mathrm{M}+1)^{+}, 319.2(\mathrm{M}+2)^{+}$in the ratio showing typical bromine isotope profile (1:1); Anal. Calcd. for $\mathrm{C}_{15} \mathrm{H}_{13} \mathrm{BrN}_{2} \mathrm{O}$ : C, 56.92; H, 4.11; N, 8.85. Found: C, 56.90; H, 4.09; N, 8.84.

N'-(4'-Methoxybenzylidene)-N-(4-methylbenzoyl)hydrazine (3k)

Yield 89.1\%; m.p.: 199-200 ${ }^{\circ} \mathrm{C}$; TLC $\mathrm{R}_{\mathrm{f}}=0.31$ [ethylacetate: hexane (3:7)]; ${ }^{1} \mathrm{H}-\mathrm{NMR}\left(400 \mathrm{MHz}, \mathrm{DMSO}-d_{6}\right): \delta=12.01\left(\mathrm{~s}, 1 \mathrm{H}, \mathrm{H}-\mathrm{N}, \mathrm{D}_{2} \mathrm{O}\right.$ exchangeable), 8.41 (s, 1H, H-7'), 7.66-7.72 (m, 4H, H-2', H-6' \& H-2, H-6), 7.27 (d, 2H, $J=7.6 \mathrm{~Hz}, \mathrm{H}-3, \mathrm{H}-5), 6.99$ (d, $2 \mathrm{H}, J=8.6 \mathrm{~Hz}, \mathrm{H}-3$ ', $\left.\mathrm{H}^{-} 5^{\prime}\right), 3.82$ (s, 3H, 4'-OCH $), 2.36$ (s, 3H, 4-CH $\left.\mathrm{CH}_{3}\right) \mathrm{ppm} ;{ }^{13} \mathrm{C}-\mathrm{NMR}(100$ $\left.\mathrm{MHz}, \mathrm{DMSO}-d_{6}\right): \delta=161.3$ (C, C-7), 159.8 (C, C-4'), 145.9 (C, C-4), 141.0 (CH, C-7'), 130.8 (C, C-1), 130.1 (CH, C-2, C-6), 128.9 (CH, C-2', C-6'), 128.6 (CH, C-3, 5), 122.4 (C, C-1'), 114.1 (CH, C-3', C-5'), 55.1 $\left(\mathrm{OCH}_{3}\right), 21.1\left(\mathrm{CH}_{3}\right)$ ppm; FT-IR $(\mathrm{KBr}): 3425(\mathrm{~N}-\mathrm{H}$ str. $), 1664(\mathrm{C}=\mathrm{O}$ str.) $\mathrm{cm}^{-1}$; MS (ESI): $\mathrm{m} / \mathrm{z}=269.1(\mathrm{M}+1)^{+}$; Anal. Calcd. for $\mathrm{C}_{16} \mathrm{H}_{16} \mathrm{~N}_{2} \mathrm{O}_{2}$ : C, 71.61; H, 5.97; N, 10.44. Found: C, 71.60; H, 5.94; N, 10.41.

N'-(4'-Methylbenzylidene)-N-(4-methylbenzoyl)hydrazine (31)

Yield $86 \%$; m.p.: $209-210^{\circ} \mathrm{C}$; TLC $\mathrm{R}_{\mathrm{f}}=0.42$ [ethylacetate: hexane (3:7)]; ${ }^{1} \mathrm{H}-\mathrm{NMR}\left(400 \mathrm{MHz}, \mathrm{DMSO}-d_{6}\right): \delta=11.85\left(\mathrm{~s}, 1 \mathrm{H}, \mathrm{H}-\mathrm{N}, \mathrm{D}_{2} \mathrm{O}\right.$ exchangeable), 8.41 (s, 1H, H-7'), 7.64-7.70 (m, 4H, H-2', H-6' \& H-2, H-6), 7.33 (d, 2H, $J=7.7 \mathrm{~Hz}, \mathrm{H}-3, \mathrm{H}-5), 7.26$ (d, 2H, $J=8.2 \mathrm{~Hz}, \mathrm{H}-3$ ', $\left.\mathrm{H}-5^{\prime}\right), 2.30$ (s, 6H, 4,4'-CH CH $_{3}$ ppm; ${ }^{13} \mathrm{C}-\mathrm{NMR}\left(100 \mathrm{MHz}, \mathrm{DMSO}-d_{6}\right): \delta=$ 161.2 (C, C-7), 143.1 (C, C-4), 140.2 (CH, C-7'), 140.1 (C, C-4'), 131.0 (C, C-1), $129.3\left(\mathrm{CH}, \mathrm{C}-2^{\prime}, \mathrm{C}^{-6}\right), 129.1\left(\mathrm{CH}, \mathrm{C}-3^{\prime}, \mathrm{C}-5^{\prime}\right), 128.8(\mathrm{CH}, \mathrm{C}-2$, C-6), 128.1 (C, C-1'), 127.6 (CH, C-3, C-5), $21.1\left(\mathrm{CH}_{3}\right)$ ppm; FT-IR
(KBr): 3429 (N-H str.), 1667 (C=O str.) $\mathrm{cm}^{-1}$; MS (ESI): $\mathrm{m} / \mathrm{z}=253.2$ $(\mathrm{M}+1)^{+}$; Anal. Calcd. for $\mathrm{C}_{16} \mathrm{H}_{16} \mathrm{~N}_{2} \mathrm{O}: \mathrm{C}, 76.13 ; \mathrm{H}, 6.34 ; \mathrm{N}, 11.10$. Found: C, $76.12 ; \mathrm{H}, 6.33 ; \mathrm{N}, 11.08$.

\section{N-Methylbenzoyl-N'-(thien-2'-yl-methylidene)hydrazine} $(3 \mathrm{~m})$

Yield $85.9 \%$; m.p.: $165-166^{\circ} \mathrm{C}$; TLC $\mathrm{R}_{\mathrm{f}}=0.30$ [ethylacetate: hexane (3:7)]; ${ }^{1} \mathrm{H}-\mathrm{NMR}\left(400 \mathrm{MHz}, \mathrm{DMSO}-d_{6}\right): \delta=11.99\left(\mathrm{~s}, 1 \mathrm{H}, \mathrm{H}-\mathrm{N}, \mathrm{D}_{2} \mathrm{O}\right.$ exchangeable), 8.70 (s, $\left.1 \mathrm{H}, \mathrm{H}-6^{\prime}\right), 7.68(\mathrm{~d}, 2 \mathrm{H}, J=7.6 \mathrm{~Hz}, \mathrm{H}-2, \mathrm{H}-6)$, 7.12-7.55 (m, 5H, H-3', H-4', H-5' \& H-3, H-5), 2.36 (s, 3H, 4-CH $)$ ppm; ${ }^{13} \mathrm{C}-\mathrm{NMR}\left(100 \mathrm{MHz}, \mathrm{DMSO}-d_{6}\right): \delta=162.9(\mathrm{C}, \mathrm{C}-7), 143.0(\mathrm{CH}$, C-6'), 140.5 (C, C-4), 138.2 (C, C-2'), 137.9 (CH, C-5'), 131.5 (C, C-1), $129.0\left(\mathrm{CH}, \mathrm{C}-4^{\prime}\right), 128.8(\mathrm{CH}, \mathrm{C}-2, \mathrm{C}-6), 127.8(\mathrm{CH}, \mathrm{C}-3$ '), $127.4(\mathrm{CH}$, C-3, C-5), $21.1\left(\mathrm{CH}_{3}\right)$ ppm; FT-IR (KBr): 3424 (N-H str.), 1666 (C=O str.) $\mathrm{cm}^{-1}$; MS (ESI): $\mathrm{m} / \mathrm{z}=245.1(\mathrm{M}+1)^{+}$; Anal. Calcd. for $\mathrm{C}_{13} \mathrm{H}_{12} \mathrm{~N}_{2} \mathrm{OS}$ : C, 63.91; H, 4.92; N, 11.47. Found: C, 63.89; H, 4.90; N, 11.46.

\section{N'-(Fur-2'-yl-methylidene)-N-(4-methylbenzoyl)hydrazine} (3n)

Yield $86.2 \%$; m.p.: $157-158^{\circ} \mathrm{C}$; TLC $\mathrm{R}_{\mathrm{f}}=0.29$ [ethylacetate: hexane (3:7)]; ${ }^{1} \mathrm{H}-\mathrm{NMR}\left(400 \mathrm{MHz}, \mathrm{DMSO}-d_{6}\right): \delta=12.01\left(\mathrm{~s}, 1 \mathrm{H}, \mathrm{H}-\mathrm{N}, \mathrm{D}_{2} \mathrm{O}\right.$ exchangeable), 8.68 (s, $\left.1 \mathrm{H}, \mathrm{H}-6^{\prime}\right), 7.66(\mathrm{~d}, 2 \mathrm{H}, J=7.7 \mathrm{~Hz}, \mathrm{H}-2, \mathrm{H}-6)$, 7.02-7.45 (m, 5H, H-3', H-4', H-5' \& H-3, H-5), 2.34 (s, 3H, 4-CH $)$ ppm; ${ }^{13} \mathrm{C}-\mathrm{NMR}\left(100 \mathrm{MHz}, \mathrm{DMSO}-d_{6}\right): \delta=162.2(\mathrm{C}, \mathrm{C}-7), 143.8(\mathrm{CH}$, C-6'), 141.1 (C, C-4), 139.1 (C, C-2'), 138.6 (CH, C-5'), 130.7 (C, C-1), $128.3(\mathrm{CH}, \mathrm{C}-2, \mathrm{C}-6), 126.9(\mathrm{CH}, \mathrm{C}-3, \mathrm{C}-5), 114.0\left(\mathrm{CH}, \mathrm{C}-4{ }^{\prime}\right), 111.6$ (CH, C-3'), $21.1\left(\mathrm{CH}_{3}\right)$ ppm; FT-IR (KBr): 3420 (N-H str.), $1663(\mathrm{C}=\mathrm{O}$ str.) $\mathrm{cm}^{-1}$; MS (ESI): $\mathrm{m} / \mathrm{z}=229.1(\mathrm{M}+1)^{+}$; Anal. Calcd. for $\mathrm{C}_{13} \mathrm{H}_{12} \mathrm{~N}_{2} \mathrm{O}_{2}$ : C, 68.39; H, 5.26; N, 12.27. Found: C, 68.38; H, 5.24; N, 12.25 .

\section{$\mathrm{N}^{\prime}-\left(1^{\prime}, 3^{\prime}\right.$ - Diphenyl-4' - pyrazolylmethylidene) - N - (4- nitrobenzoyl)hydrazine (3o)}

Yield 87.6 \%; m.p.: 232-233 ${ }^{\circ} \mathrm{C}$; TLC $_{\mathrm{f}}=0.33$ [ethylacetate: hexane (3:7)]; ${ }^{1} \mathrm{H}-\mathrm{NMR}\left(400 \mathrm{MHz}, \mathrm{DMSO}-d_{6}\right): \delta=11.98\left(\mathrm{~s}, 1 \mathrm{H}, \mathrm{H}-\mathrm{N}, \mathrm{D}_{2} \mathrm{O}\right.$ exchangeable), 8.98 (s, $\left.1 \mathrm{H}, \mathrm{H}-5^{\prime}\right), 8.61\left(\mathrm{~s}, 1 \mathrm{H}, \mathrm{H}-6^{\prime}\right), 8.16-8.36(\mathrm{~m}, 4 \mathrm{H}$, H-2, H-6 \& H-3, H-5), 8.02 (d, 2H, $J=8.0$ Hz, H-2", H-6"), 7.75 (d, $2 \mathrm{H}, J=7.2 \mathrm{~Hz}, \mathrm{H}-2$ "', H-6"'), 7.35-7.56 (m, 6H, H-Ph" \& H-Ph"') ppm; ${ }^{13} \mathrm{C}-\mathrm{NMR}\left(100 \mathrm{MHz}, \mathrm{DMSO}-d_{6}\right): \delta=160.9$ (C, C-7), 152.0 (C, C-3'), 149.1 (C, C-4), 142.2 (CH, C-6'), 139.1 (C, C-1"), 139.0 (C, C-1), 132.0 (C, C-1"'), 129.4 (CH, C-3"', C-5"'), 129.0 (CH, C-3", C-5"), $128.6(\mathrm{CH}$, C-2, C-6), 128.5 (CH, C-4"'), 128.4 (CH, C-2"', C-6"'), 127.0 (CH, C-5'), 126.8 (CH, C-4"), 123.4 (CH, C-3, C-5), 118.7 (CH, C-2", C-6"), 116.6 (C, C-4') ppm; FT-IR (KBr): 3434 (N-H str.), 1670 (C=O str.) cm ${ }^{-1}$; MS (ESI): $\mathrm{m} / \mathrm{z}=412.1(\mathrm{M}+1)^{+}$; Anal. Calcd. for $\mathrm{C}_{23} \mathrm{H}_{17} \mathrm{~N}_{5} \mathrm{O}_{3}: \mathrm{C}, 67.14 ; \mathrm{H}$, 4.13; N, 17.03. Found: C, 67.10; H, 4.12; N, 17.01.

\section{N-(4-Nitrobenzoyl)-N'-(4'-nitrobenzylidene)hydrazine (3p)}

Yield $88.5 \%$; m.p.: $247-248^{\circ} \mathrm{C}$; $\mathrm{TLC}_{\mathrm{f}}=0.36$ [ethylacetate: hexane (3:7)]; ${ }^{1} \mathrm{H}-\mathrm{NMR}\left(400 \mathrm{MHz}, \mathrm{DMSO}-d_{6}\right): \delta=12.10\left(\mathrm{~s}, 1 \mathrm{H}, \mathrm{H}-\mathrm{N}, \mathrm{D}_{2} \mathrm{O}\right.$ exchangeable), 8.67 (s, 1H, H-7'), 8.05-8.38 (m, 8H, H-Ph \& H-Ph') ppm; ${ }^{13} \mathrm{C}-\mathrm{NMR}\left(100 \mathrm{MHz}, \mathrm{DMSO}-d_{6}\right): \delta=163.4(\mathrm{C}, \mathrm{C}-7), 148.3(\mathrm{C}$, C-4), 148.1 (C, C-4'), 141.9 (C, C-1'), 140.6 (CH, C-7'), 131.4 (C, C-1), 128.0 (CH, C-2, C-6), 127.9 (CH, C-2', C-6'), 123.7 (CH, C-3', C-5'), 123.6 (CH, C-3, C-5) ppm; FT-IR (KBr): 3436 (N-H str.), 1668 (C=O str.), 1546 ( $\mathrm{NO}_{2}$ asymmetric str.), $1354\left(\mathrm{NO}_{2}\right.$ symmetric str.) $\mathrm{cm}^{-1}$; MS (ESI): $\mathrm{m} / \mathrm{z}=315.1(\mathrm{M}+1)^{+}$; Anal. Calcd. for $\mathrm{C}_{14} \mathrm{H}_{10} \mathrm{~N}_{4} \mathrm{O}_{5}: \mathrm{C}, 53.49 ; \mathrm{H}$, 3.18; N, 17.83. Found: C, 53.47; H, 3.16; N, 17.81 .

\section{N'-(4'-Bromobenzylidene)-N-4-nitrobenzoylhydrazine (3q)}

Yield $86.5 \%$; m.p.: $227-228^{\circ} \mathrm{C}$; $\mathrm{TLC}_{\mathrm{f}}=0.48$ [ethylacetate: hexane 
Citation: Kumar V, Kumar M, Beniwal V, Gupta GK, Kumar S, et al. (2016) Synthesis of Some Aroylhydrazones and 2,5-Disubstituted-1,3,4Oxadiazoles as DNA Photocleaving Agents. Med chem (Los Angeles) 6: 474-485. doi:10.4172/2161-0444.1000386

(3:7)]; ${ }^{1} \mathrm{H}-\mathrm{NMR}\left(400 \mathrm{MHz}, \mathrm{DMSO}-d_{6}\right): \delta=12.14\left(\mathrm{~s}, 1 \mathrm{H}, \mathrm{H}-\mathrm{N}, \mathrm{D}_{2} \mathrm{O}\right.$ exchangeable), 8.47 (s, 1H, H-7'), 8.17-8.36 (m, 4H, H-2, H-6 \& H-3, $\mathrm{H}-5)$, 7.51-7.71 (m, 4H, H-Ph') ppm; ${ }^{13} \mathrm{C}-\mathrm{NMR}\left(100 \mathrm{MHz}, \mathrm{DMSO}-d_{6}\right)$ : $\delta=163.4(\mathrm{C}, \mathrm{C}-7), 149.1(\mathrm{C}, \mathrm{C}-4), 141.0\left(\mathrm{CH}, \mathrm{C}-7^{\prime}\right), 133.5\left(\mathrm{CH}, \mathrm{C}-3^{\prime}\right.$, C-5'), 131.2 (C, C-1), 130.0 (C, C-1'), 127.7 (CH, C-2, C-6), $127.6(\mathrm{CH}$, C-2', C-6'), $124.2(\mathrm{CH}, \mathrm{C}-3, \mathrm{C}-5), 123.4(\mathrm{C}, \mathrm{C}-4$ ') ppm; FT-IR (KBr): 3435 (N-H str.), 1669 (C=O str.) $\mathrm{cm}^{-1}$; MS (ESI): $\mathrm{m} / \mathrm{z}=348.1(\mathrm{M}+1)^{+}$, $350.2(\mathrm{M}+2)^{+}$in the ratio showing typical bromine isotope profile $(1: 1)$; Anal. Calcd. for $\mathrm{C}_{14} \mathrm{H}_{10} \mathrm{BrN}_{3} \mathrm{O}_{3}$ : C, 48.40; $\mathrm{H}, 2.88 ; \mathrm{N}, 12.10$. Found: C, $48.38 ; \mathrm{H}, 2.86 ; \mathrm{N}, 12.09$.

\section{N'-(4'-Methoxybenzylidene)-N-(4-nitrobenzoyl)hydrazine} (3r)

Yield 89.2 \%; m.p.: 198- $199^{\circ} \mathrm{C}$; TLC $\mathrm{R}_{\mathrm{f}}=0.33$ [ethylacetate: hexane (3:7)]; ${ }^{1} \mathrm{H}-\mathrm{NMR}\left(400 \mathrm{MHz}, \mathrm{DMSO}-d_{6}\right): \delta=11.96\left(\mathrm{~s}, 1 \mathrm{H}, \mathrm{H}-\mathrm{N}, \mathrm{D}_{2} \mathrm{O}\right.$ exchangeable), 8.40 (s, 1H, H-7'), 8.14-8.33 (m, $4 \mathrm{H}, \mathrm{H}-2, \mathrm{H}-6$ \& H-3, H-5), 7.68 (d, 2H, J = 8.6 Hz, H-2', H-6'), 6.97 (d, 2H, $J=8.7 \mathrm{~Hz}, \mathrm{H}-3$ ', H-5'), 3.80 (s, 3H, 4'-OCH $)$ ppm; ${ }^{13} \mathrm{C}-\mathrm{NMR}\left(100 \mathrm{MHz}, \mathrm{DMSO}-d_{6}\right)$ : $\delta=161.5(\mathrm{C}, \mathrm{C}-7), 160.3\left(\mathrm{C}, \mathrm{C}-4^{\prime}\right), 148.8(\mathrm{C}, \mathrm{C}-4), 139.1\left(\mathrm{CH}, \mathrm{C}-7^{\prime}\right)$, 130.6 (C, C-1), 128.8 (CH, C-2', C-6'), $126.5(\mathrm{CH}, \mathrm{C}-2, \mathrm{C}-6), 123.3(\mathrm{CH}$, C-3, C-5), 122.5 (C, C-1'), $114.1\left(\mathrm{CH}, \mathrm{C}-3^{\prime}, \mathrm{C}-5^{\prime}\right), 55.1\left(\mathrm{OCH}_{3}\right) \mathrm{ppm}$; FT-IR (KBr): 3426 (N-H str.), 1670 (C=O str.) $\mathrm{cm}^{-1}$; MS (ESI): $\mathrm{m} / \mathrm{z}=$ $300.1(\mathrm{M}+1)^{+}$; Anal. Calcd. for $\mathrm{C}_{15} \mathrm{H}_{13} \mathrm{~N}_{3} \mathrm{O}_{4}$ : C, 60.18; $\mathrm{H}, 4.35 ; \mathrm{N}, 14.04$. Found: $\mathrm{C}, 60.16 ; \mathrm{H}, 4.33 ; \mathrm{N}, 14.03$.

\section{N'-(4'-Methylbenzylidene)-N-(4-nitrobenzoyl)hydrazine (3s)}

Yield $86.7 \%$; m.p.: $215-216^{\circ} \mathrm{C}$; TLC $\mathrm{R}_{\mathrm{f}}=0.45$ [ethylacetate: hexane (3:7)]; ${ }^{1} \mathrm{H}-\mathrm{NMR}\left(400 \mathrm{MHz}, \mathrm{DMSO}-d_{6}\right): \delta=12.05$ (s, $1 \mathrm{H}, \mathrm{H}-\mathrm{N}, \mathrm{D}_{2} \mathrm{O}$ exchangeable), 8.44 (s, 1H, H-7'), 8.16-8.36 (m, 4H, H-2, H-6 \& H-3, H-5), 7.65 (d, 2H, $\left.\left.J=8.6 \mathrm{~Hz}, \mathrm{H}-2^{\prime}, \mathrm{H}^{-6}\right)^{\prime}\right), 7.25$ (d, 2H, $J=8.4 \mathrm{~Hz}, \mathrm{H}-3^{\prime}$, H-5'), 2.32 (s, 3H, 4'-CH $)$ ppm; ${ }^{13} \mathrm{C}-\mathrm{NMR}\left(100 \mathrm{MHz}, \mathrm{DMSO}-d_{6}\right): \delta$ $=161.4(\mathrm{C}, \mathrm{C}-7), 149.1(\mathrm{C}, \mathrm{C}-4), 140.1\left(\mathrm{CH}, \mathrm{C}-7^{\prime}\right), 139.0\left(\mathrm{C}, \mathrm{C}-4^{\prime}\right)$, 130.6 (C, C-1), 129.3 (CH, C-2', C-6'), 129.0 (CH, C-3', C-5'), 128.2 (C, C-1'), 127.2 (CH, C-2, C-6), $123.3(\mathrm{CH}, \mathrm{C}-3, \mathrm{C}-5), 21.1\left(\mathrm{CH}_{3}\right) \mathrm{ppm}$; FT-IR (KBr): 3428 (N-H str.), 1667 (C=O str.) $\mathrm{cm}^{-1}$; MS (ESI): $\mathrm{m} / \mathrm{z}=$ $284.2(\mathrm{M}+1)^{+}$; Anal. Calcd. for $\mathrm{C}_{15} \mathrm{H}_{13} \mathrm{~N}_{3} \mathrm{O}_{3}: \mathrm{C}, 63.56 ; \mathrm{H}, 4.59 ; \mathrm{N}, 14.83$. Found: $\mathrm{C}, 63.55 ; \mathrm{H}, 4.57 ; \mathrm{N}, 14.81$.

\section{N-4-Nitrobenzoyl-N'-(thien-2'-yl-methylidene)hydrazine} (3t)

Yield $85.7 \%$; m.p.: $189-190^{\circ} \mathrm{C}$; TLC $\mathrm{R}_{\mathrm{f}}=0.30$ [ethylacetate: hexane (3:7)]; ${ }^{1} \mathrm{H}-\mathrm{NMR}\left(400 \mathrm{MHz}, \mathrm{DMSO}-d_{6}\right): \delta=12.04\left(\mathrm{~s}, 1 \mathrm{H}, \mathrm{H}-\mathrm{N}, \mathrm{D}_{2} \mathrm{O}\right.$ exchangeable), 8.67 (s, 1H, H-6'), 8.12-8.33 (m, 4H, H-2, H-6 \& H-3, $\mathrm{H}-5)$, 7.09-7.58 (m, 3H, H-2', H-3', H-5') ppm; ${ }^{13} \mathrm{C}-\mathrm{NMR}(100 \mathrm{MHz}$, DMSO- $\left.d_{6}\right): \delta=161.2$ (C, C-7), 149.1 (C, C-4), 143.9 (CH, C-6'), 138.9 (C, C-2'), 138.7 (CH, C-5'), 130.9 (C, C-1), 129.0 (CH, C-2, C-6), 128.9 (CH, C-4'), 127.5 (CH, C-3'), $123.3(\mathrm{CH}, \mathrm{C}-3, \mathrm{C}-5)$ ppm; FT-IR (KBr): 3425 (N-H str.), 1667 (C=O str.) $\mathrm{cm}^{-1}$; MS (ESI): $\mathrm{m} / \mathrm{z}=276.2(\mathrm{M}+1)^{+}$; Anal. Calcd. for $\mathrm{C}_{12} \mathrm{H}_{9} \mathrm{~N}_{3} \mathrm{O}_{3} \mathrm{~S}$ : C, 52.32; $\mathrm{H}, 3.27 ; \mathrm{N}, 15.26$. Found: $\mathrm{C}$, $52.31 ; \mathrm{H}, 3.24 ; \mathrm{N}, 15.24$.

N'-(Fur-2'-yl-methylidene)-N-(4-nitrobenzoyl)hydrazine (3u)

Yield $84.9 \%$; m.p.: $168-169^{\circ} \mathrm{C}$; TLC $\mathrm{R}_{\mathrm{f}}=0.27$ [ethylacetate: hexane (3:7)]; ${ }^{1} \mathrm{H}-\mathrm{NMR}$ (400 MHz, DMSO- $d_{6}$ ): $\delta=12.06\left(\mathrm{~s}, 1 \mathrm{H}, \mathrm{H}-\mathrm{N}, \mathrm{D}_{2} \mathrm{O}\right.$ exchangeable), 8.38 (s, 1H, H-6'), 8.14-8.36 (m, 4H, H-2, H-6 \& H-3, H-5), 6.58-7.74 (m, 3H, H-2', H-3', H-5') ppm; ${ }^{13} \mathrm{C}-\mathrm{NMR}(100 \mathrm{MHz}$, DMSO- $\left.d_{6}\right): \delta=161.3$ (C, C-7), $149.2(\mathrm{C}, \mathrm{C}-4), 144.9$ (CH, C-6'), 138.8 (C, C-2'), 138.5 (CH, C-5'), 130.8 (C, C-1), 129.0 (CH, C-2, C-6), 123.3 (CH, C-3, C-5), $113.6(\mathrm{CH}, \mathrm{C}-4$ '), $111.9(\mathrm{CH}, \mathrm{C}-3$ ') ppm; FT-IR (KBr):
3426 (N-H str.), 1666 (C=O str.) $\mathrm{cm}^{-1}$; MS (ESI): $\mathrm{m} / \mathrm{z}=260.0(\mathrm{M}+1)^{+}$; Anal. Calcd. for $\mathrm{C}_{12} \mathrm{H}_{9} \mathrm{~N}_{3} \mathrm{O}_{4}: \mathrm{C}, 55.60 ; \mathrm{H}, 3.47 ; \mathrm{N}, 16.22$. Found: C, $55.59 ; \mathrm{H}, 3.45 ; \mathrm{N}, 16.19$.

\section{Synthesis of 2,5-disubstituted-1,3,4-oxadiazoles (4)}

\section{General procedure}

IBD $(0.011 \mathrm{~mol})$ was added in a portion-wise manner to the suspension or solution of an appropriate aroylhydrazone (3, 0.01 $\mathrm{mol}$ ) in dichloromethane while stirring. The reaction mass was further stirred for $0.5-2.15 \mathrm{~h}$ and the reaction was monitored by TLC. After completion of the reaction, the solvent was evaporated and residue was triturated with petroleum ether twice to obtain the crude product 4 which was recrystallised from ethanol [36].

\section{5-(1",3"-Diphenyl-4"-pyrazolyl)-2-phenyl-1,3,4-oxadiazole} (4a)

Yield 90 \%; m.p.: (Obs.) $136-137^{\circ} \mathrm{C}$, m.p.: (Lit.) $136-138^{\circ} \mathrm{C}$; TLC $\mathrm{R}_{\mathrm{f}}=0.61$ [ethylacetate: hexane (3:7)]; ${ }^{1} \mathrm{H}-\mathrm{NMR}\left(400 \mathrm{MHz}, \mathrm{CDCl}_{3}\right): \delta=$ 8.71 (s, 1H, H-5"), 7.92-7.97 (m, 4H, H-2"'", H-6"' \& H-2', H-6'), 7.84 (d, $2 \mathrm{H}, J=7.6 \mathrm{~Hz}, \mathrm{H}-2^{\prime \prime \prime}, \mathrm{H}-6$ "') $^{\prime}$, 7.26-7.55 (m, 9H, H-3', H-4', H-5', H-Ph"' \& $\left.\mathrm{H}-\mathrm{Ph}^{\prime \prime \prime}\right) \mathrm{ppm} ;{ }^{13} \mathrm{C}-\mathrm{NMR}\left(100 \mathrm{MHz}, \mathrm{CDCl}_{3}\right): \delta=164.0$ (C, C-5), 159.9 (C, C-2), 152.0 (C, C-3"), 139.2 (C, C-1"'), 131.8 (C, C-1"'), 131.6 (CH, C-5"), 129.7 (CH, C-3"'", C-5"''), 129.4 (CH, C-4'), 129.1 (CH, C-3"', C-5"'), 129.0 (CH, C-4"'), 128.8 (CH, C-2"'", C-6"'"), 128.3 (CH, C-2', C-6'), 127.6 (CH, C-4'"), 126.8 (CH, C-3', C-5'), 123.8 (C, C-1'), 119.5 (CH, C-2"', C-6"'), 106.5 (C, C-4") ppm; FT-IR (KBr): transparent in the region of (N-H str.) and ( $\mathrm{C}=\mathrm{O}$ str.), 1250 (C-O str.) $\mathrm{cm}^{-1}$; MS (ESI): $\mathrm{m} / \mathrm{z}=365.2(\mathrm{M}+1)^{+}$; Anal. Calcd. for $\mathrm{C}_{23} \mathrm{H}_{16} \mathrm{~N}_{4} \mathrm{O}: \mathrm{C}, 75.78 ; \mathrm{H}, 4.39 ; \mathrm{N}$, 15.38. Found: C, 75.75; H, 4.37; N, 15.34 .

\section{5-(4"-Nitrophenyl)-2-phenyl-1,3,4-oxadiazole (4b)}

Yield $88.5 \%$; m.p.: (Obs.) $209-210^{\circ} \mathrm{C}$, m.p.: (Lit.) $209-210^{\circ} \mathrm{C}$; TLC $\mathrm{R}_{\mathrm{f}}=0.75$ [ethylacetate: hexane (3:7)]; ${ }^{1} \mathrm{H}-\mathrm{NMR}\left(400 \mathrm{MHz}\right.$, DMSO- $\left.d_{6}\right)$ : $\delta=8.33-8.48$ (m, 4H, H-Ph"), 7.35-7.75 (m, 5H, H-Ph') ppm; ${ }^{13} \mathrm{C}-\mathrm{NMR}$ $\left(100 \mathrm{MHz}, \mathrm{DMSO}-d_{6}\right): \delta=162.1$ (C, C-5), 160.7 (C, C-2), $148.9(\mathrm{C}$, C-4"), 130.1 (CH, C-4'), 129.1 (C, C-1"), 128.7 (CH, C-2', C-6'), 127.0 (CH, C-3', C-5'), 126.5 (CH, C-2", C-6"), 124.2 (CH, C-3", C-5"), 123.9 $\left(\mathrm{CH}, \mathrm{C}, \mathrm{C}-1^{\prime}\right)$ ppm; FT-IR $(\mathrm{KBr})$ : transparent in the region of $(\mathrm{N}-\mathrm{H}$ str.) and ( $\mathrm{C}=\mathrm{O}$ str.), $1543\left(\mathrm{NO}_{2}\right.$ asymmetric str.), $1349\left(\mathrm{NO}_{2}\right.$ symmetric str.), 1252 (C-O str.) $\mathrm{cm}^{-1}$; MS (ESI): $\mathrm{m} / \mathrm{z}=268.2(\mathrm{M}+1)^{+}$; Anal. Calcd. for $\mathrm{C}_{14} \mathrm{H}_{9} \mathrm{~N}_{3} \mathrm{O}_{3}: \mathrm{C}, 62.87 ; \mathrm{H}, 3.37 ; \mathrm{N}, 15.72$. Found: $\mathrm{C}, 62.85 ; \mathrm{H}, 3.34 ; \mathrm{N}$, 15.71 .

\section{5-(4"-Bromophenyl)-2-phenyl-1,3,4-oxadiazole (4c)}

Yield $88.1 \%$; m.p.: (Obs.) $167-168^{\circ} \mathrm{C}$, m.p.: (Lit.) $167^{\circ} \mathrm{C}$; TLC $\mathrm{R}_{\mathrm{f}}=$ 0.81 [ethylacetate: hexane (3:7)]; ${ }^{1} \mathrm{H}-\mathrm{NMR}\left(400 \mathrm{MHz}, \mathrm{DMSO}-d_{6}\right): \delta=$ 8.01 (d, 2H, $J=7.2 \mathrm{~Hz}, \mathrm{H}-2^{\prime}, \mathrm{H}^{-6}$ ), $7.38-7.80$ (m, 7H, H-3', H-4', H-5 \& H-Ph") ppm; ${ }^{13} \mathrm{C}-\mathrm{NMR}\left(100 \mathrm{MHz}, \mathrm{DMSO}-d_{6}\right): \delta=163.5(\mathrm{C}, \mathrm{C}-5)$, 160.9 (C, C-2), 132.5 (CH, C-3", C-5"), 129.8 (CH, C-4'), $128.9(\mathrm{CH}$, C-2', C-6'), 128.1 (CH, C-2", C-6"), 126.8 (CH, C-3', C-5'), 126.5 (C, C-1"), 124.0 (C, C-1'), 123.0 (C, C-4") ppm; FT-IR (KBr): transparent in the region of $(\mathrm{N}-\mathrm{H}$ str. $)$ and $(\mathrm{C}=\mathrm{O}$ str. $), 1245(\mathrm{C}-\mathrm{O}$ str. $) \mathrm{cm}^{-1}$; MS (ESI): $\mathrm{m} / \mathrm{z}=301.1(\mathrm{M}+1)^{+}, 303.1(\mathrm{M}+2)^{+}$in the ratio showing typical bromine isotope profile (1:1); Anal. Calcd. for $\mathrm{C}_{14} \mathrm{H}_{9} \mathrm{BrN}_{2} \mathrm{O}: \mathrm{C}, 55.98 ; \mathrm{H}, 3.00 ; \mathrm{N}$, 9.33. Found: C, 55.96; H, 2.98; N, 9.32.

\section{5-(4"-Methoxyphenyl)-2-phenyl-1,3,4-oxadiazole (4d)}

Yield 91 \%; m.p.: (Obs.) $150-151{ }^{\circ} \mathrm{C}$, m.p.: (Lit.) $150{ }^{\circ} \mathrm{C}$; $\mathrm{TLC} \mathrm{R}_{\mathrm{f}}=$ 0.73 [ethylacetate: hexane (3:7)]; ${ }^{1} \mathrm{H}-\mathrm{NMR}\left(400 \mathrm{MHz}, \mathrm{CDCl}_{3}\right): \delta=7.35$ 8.10 (m, 7H, H-2", H-6" \& H-Ph'), 7.12 (d, 2H, J = 8.5 Hz, H-3", H-5"), 
Citation: Kumar V, Kumar M, Beniwal V, Gupta GK, Kumar S, et al. (2016) Synthesis of Some Aroylhydrazones and 2,5-Disubstituted-1,3,4Oxadiazoles as DNA Photocleaving Agents. Med chem (Los Angeles) 6: 474-485. doi:10.4172/2161-0444.1000386

$3.84\left(\mathrm{~s}, 3 \mathrm{H}, 4\right.$ "-OCH $\left.{ }_{3}\right) \mathrm{ppm} ;{ }^{13} \mathrm{C}-\mathrm{NMR}\left(100 \mathrm{MHz}, \mathrm{DMSO}-d_{6}\right): \delta=162.4$ (C, C-5), 160.1 (C, C-2), 159.7 (C, C-4"), $130.0(\mathrm{CH}, \mathrm{C}-4$ '), $128.7(\mathrm{CH}$, C-2", C-6"), $128.4(\mathrm{CH}, \mathrm{C}-2$ ', C-6'), $126.9(\mathrm{CH}, \mathrm{C}-3$ ', C-5'), $124.2(\mathrm{C}$, C-1'), 120.8 (C, C-1"), 114.8 (CH, C-3", C-5"), $55.3\left(\mathrm{OCH}_{3}\right)$ ppm; FTIR ( $\mathrm{KBr})$ : transparent in the region of (N-H str.) and ( $\mathrm{C}=\mathrm{O}$ str.), 1249 (C-O str.) $\mathrm{cm}^{-1}$; MS (ESI): $\mathrm{m} / \mathrm{z}=253.2(\mathrm{M}+1)^{+}$; Anal. Calcd. for $\mathrm{C}_{15} \mathrm{H}_{12}$ $\mathrm{N}_{2} \mathrm{O}_{2}$ : C, 71.37; H, 4.76; N, 11.10. Found: C, 71.36; H, 4.75; N, 11.09 .

\section{5-(4"-Methylphenyl)-2-phenyl-1,3,4-oxadiazole (4e)}

Yield $90.2 \%$; m.p.: (Obs.) $148-149^{\circ} \mathrm{C}$, m.p.: (Lit.) $148^{\circ} \mathrm{C}$; $\mathrm{TLC} \mathrm{R}_{\mathrm{f}}=$ 0.77 [ethylacetate: hexane (3:7)]; ${ }^{1} \mathrm{H}-\mathrm{NMR}\left(400 \mathrm{MHz}, \mathrm{CDCl}_{3}\right): \delta=7.45$ 8.05 (m, 7H, H-2", H-6" \& H-Ph'), 7.28 (d, 2H, $J=8.4$ Hz, H-3", H-5"), $2.40\left(\mathrm{~s}, 3 \mathrm{H}, 4\right.$ "- $\left.\mathrm{CH}_{3}\right) \mathrm{ppm} ;{ }^{13} \mathrm{C}-\mathrm{NMR}\left(100 \mathrm{MHz}, \mathrm{CDCl}_{3}\right): \delta=165.4(\mathrm{C}$, C-5), 160.5 (C, C-2), 144.2 (C, C-4"), 130.2 (CH, C-2", C-6"), 129.7 (CH, C-4'), 128.6 (CH, C-2', C-6'), 127.1 (CH, C-3', C-5'), $127.0(\mathrm{CH}, \mathrm{C}-3 "$, C-5"), 123.9 (C, C-1'), 120.3 (C, C-1"), $21.7\left(\mathrm{CH}_{3}\right)$ ppm; FT-IR ( $\left.\mathrm{KBr}\right)$ : transparent in the region of (N-H str.) and (C=O str.), 1253 (C-O str.) $\mathrm{cm}^{-1}$; MS (ESI): $\mathrm{m} / \mathrm{z}=237.3(\mathrm{M}+1)^{+}$; Anal. Calcd. for $\mathrm{C}_{15} \mathrm{H}_{12} \mathrm{~N}_{2} \mathrm{O}: \mathrm{C}$, 76.17; H, 5.08; N, 11.85. Found: C, 76.16; H, 5.05; N, 11.84 .

\section{2-Phenyl-5-(thien-2"-yl)-1,3,4-oxadiazole (4f)}

Yield $87.9 \%$; m.p.: $110-111^{\circ} \mathrm{C}$; $\mathrm{TLC}_{\mathrm{f}}=0.75$ [ethylacetate: hexane (3:7)]; ${ }^{1} \mathrm{H}-\mathrm{NMR}\left(400 \mathrm{MHz}, \mathrm{CDCl}_{3}\right): \delta=8.12(\mathrm{~d}, 2 \mathrm{H}, J=6.9 \mathrm{~Hz}, \mathrm{H}-2$, H-6'), 7.18-7.84 (m, 6H, H-3', H-4', H-5' \& H-3", H-4", H-5") ppm; ${ }^{13} \mathrm{C}-\mathrm{NMR}\left(100 \mathrm{MHz}, \mathrm{CDCl}_{3}\right): \delta=164.0$ (C, C-5), 160.9 (C, C-2), 131.8 (CH, C-5"), 130.2 (CH, C-3"), 129.8 (CH, C-4'), 129.1 (CH, C-2', C-6'), 128.2 (CH, C-4"), 127.0 (CH, C-3', C-5'), 125.2 (C, C-2"), 123.7 (C, $\left.\mathrm{C}-1^{\prime}\right)$ ppm; FT-IR (KBr): transparent in the region of (N-H str.) and (C=O str.), 1246 (C-O str.) $\mathrm{cm}^{-1}$; MS (ESI): $\mathrm{m} / \mathrm{z}=229.0(\mathrm{M}+1)^{+}$; Anal. Calcd. for $\mathrm{C}_{12} \mathrm{H}_{8} \mathrm{~N}_{2} \mathrm{OS}$ : C, 63.16; $\mathrm{H}, 3.51 ; \mathrm{N}, 12.28$. Found: $\mathrm{C}, 63.14 ; \mathrm{H}$, $3.48 ; \mathrm{N}, 12.26$

\section{5-(Fur-2"-yl)-2-phenyl-1,3,4-oxadiazole (4g)}

Yield 85.9 \%; m.p.: (Obs.) $103-104{ }^{\circ} \mathrm{C}$, m.p.: (Lit.) $103{ }^{\circ} \mathrm{C}$; TLC $\mathrm{R}_{f}$ $=0.64$ [ethylacetate: hexane (3:7)]; ${ }^{1} \mathrm{H}-\mathrm{NMR}\left(400 \mathrm{MHz}, \mathrm{CDCl}_{3}\right): \delta=$ 8.10 (d, $2 \mathrm{H}, J=7.0 \mathrm{~Hz}, \mathrm{H}-2^{\prime}, \mathrm{H}^{-6}$ ) $)$ 7.12-7.88 (m, 6H, H-3', H-4', H-5' \& H-3", H-4", H-5") ppm; ${ }^{13} \mathrm{C}-\mathrm{NMR}\left(100 \mathrm{MHz}, \mathrm{DMSO}-d_{6}\right): \delta=164.3$ (C, C-5), 159.8 (C, C-2), 133.4 (CH, C-5"), $130.1(\mathrm{CH}, \mathrm{C}-4$ '), 128.8 (CH, C-2', C-6'), 128.5 (C, C-2"), 127.1 (CH, C-3', C-5'), 123.9 (C, C-1'), 114.8 (CH, C-3"), $114.5(\mathrm{CH}, \mathrm{C}-4 ")$ ppm; FT-IR (KBr): transparent in the region of (N-H str.) and (C=O str.), $1244\left(\mathrm{C}-\mathrm{O}\right.$ str. $\mathrm{cm}^{-1}$; MS (ESI): $\mathrm{m} / \mathrm{z}=213.1(\mathrm{M}+1)^{+}$; Anal. Calcd. for $\mathrm{C}_{12} \mathrm{H}_{8} \mathrm{~N}_{2} \mathrm{O}_{2}: \mathrm{C}, 67.89 ; \mathrm{H}, 3.77 ; \mathrm{N}$, 13.20. Found: C, $67.86 ; \mathrm{H}, 3.75 ; \mathrm{N}, 13.18$.

\section{5-(1",3"-Diphenyl-4"-pyrazolyl)-2-(4'-methylphenyl)-1,3,4- oxadiazole (4h)}

Yield $88.6 \%$; m.p.: $145-146^{\circ} \mathrm{C}$; TLC $\mathrm{R}_{\mathrm{f}}=0.57$ [ethylacetate: hexane (3:7)]; ${ }^{1} \mathrm{H}-\mathrm{NMR}\left(400 \mathrm{MHz}, \mathrm{CDCl}_{3}\right.$ ): $\delta=8.61$ (s, $\left.1 \mathrm{H}, \mathrm{H}-5 "\right)$, 7.75-7.87 (m, 6H, H-2"', H-6"', H-2"', H-6"' \& H-2', H-6'), 7.18-7.47 (m, 8H, H-3', H-5', H-Ph"' \& H-Ph"'), 2.34 (s, 3H, 4'-CH $)$ ppm; ${ }^{13} \mathrm{C}-\mathrm{NMR}(100 \mathrm{MHz}$, $\left.\mathrm{CDCl}_{3}\right): \delta=163.9$ (C, C-5), $162.3(\mathrm{C}, \mathrm{C}-2), 151.9$ (C, C-3"), $142.3(\mathrm{C}$, C-4'), 139.3 (C, C-1"'), 132.0 (C, C-1'"'), 131.7 (CH, C-5"), 129.6 (CH, C-2', C-6'), 129.5 (CH, C-3"'", C-5'"'), 129.2 (CH, C-3"', C-5"'), 128.9 (CH, C-4"''), 128.8 (CH, C-2"'", C-6"'"), $127.6(\mathrm{CH}, \mathrm{C}-3$ ', C-5'), 127.3 (CH, C-4"'), 120.5 (C, C-1'), 119.4 (CH, C-2"', C-6"'), 106.5 (C, C-4"), $21.3\left(\mathrm{CH}_{3}\right)$ ppm; FT-IR $(\mathrm{KBr})$ : transparent in the region of (N-H str.) and $\left(\mathrm{C}=\mathrm{O}\right.$ str.), 1252 (C-O str.) $\mathrm{cm}^{-1}$; MS (ESI): $\mathrm{m} / \mathrm{z}=379.1(\mathrm{M}+1)^{+}$; Anal. Calcd. for $\mathrm{C}_{24} \mathrm{H}_{18} \mathrm{~N}_{4} \mathrm{O}$ : C, 76.17; H, 4.76; N, 14.81. Found: C, 76.16; H, 4.74; N, 14.79 .

\section{2-(4'-Methylphenyl)-5-(4"-Nitrophenyl)-1,3,4-oxadiazole (4i)}

Yield $90.2 \%$; m.p.: $168-169^{\circ} \mathrm{C}$; $\mathrm{TLC}_{\mathrm{f}}=0.69$ [ethylacetate: hexane (3:7)]; ${ }^{1} \mathrm{H}-\mathrm{NMR}\left(400 \mathrm{MHz}, \mathrm{DMSO}-d_{6}\right): \delta=8.36-8.44(\mathrm{~m}, 4 \mathrm{H}, \mathrm{H}-\mathrm{Ph} ")$, 8.03 (d, $\left.2 \mathrm{H}, J=8.2 \mathrm{~Hz}, \mathrm{H}-2^{\prime}, \mathrm{H}^{\prime} 6^{\prime}\right), 7.41$ (d, $\left.2 \mathrm{H}, J=8.1 \mathrm{~Hz}, \mathrm{H}-3^{\prime}, \mathrm{H}-5^{\prime}\right)$, $2.34\left(\mathrm{~s}, 3 \mathrm{H}, 44^{\prime}-\mathrm{CH}_{3}\right)$ ppm; ${ }^{13} \mathrm{C}-\mathrm{NMR}\left(100 \mathrm{MHz}, \mathrm{DMSO}-d_{6}\right): \delta=164.9$ (C, C-2), 162.3 (C, C-5), 149.0 (C, C-4"), 142.5 (C, C-4'), 129.8 (CH, C-2', C-6'), 129.0 (C, C-1"), 127.8 (CH, C-3', C-5'), 126.7 (CH, C-2", C-6"), 124.4 (CH, C-3", C-5"), 120.2 (C, C-1'), $21.2\left(\mathrm{CH}_{3}\right)$ ppm; FTIR ( $\mathrm{KBr})$ : transparent in the region of (N-H str.) and (C=O str.), 1545 ( $\mathrm{NO}_{2}$ asymmetric str.), 1347 ( $\mathrm{NO}_{2}$ symmetric str.), 1255 (C-O str.) $\mathrm{cm}^{-1}$; MS (ESI): $\mathrm{m} / \mathrm{z}=282.1(\mathrm{M}+1)^{+}$; Anal. Calcd. for $\mathrm{C}_{15} \mathrm{H}_{11} \mathrm{~N}_{3} \mathrm{O}_{3}: \mathrm{C}, 64.03$; $\mathrm{H}, 3.91 ; \mathrm{N}, 14.94$. Found: C, 64.02; H, 3.90; N, 14.92 .

\section{5-(4"-Bromophenyl)-2-(4'-methylphenyl)-1,3,4-oxadiazole} (4j)

Yield $87 \%$; m.p.: $205-206^{\circ} \mathrm{C}$; TLC $\mathrm{R}_{\mathrm{f}}=0.75$ [ethylacetate: hexane (3:7)]; ${ }^{1} \mathrm{H}-\mathrm{NMR}\left(400 \mathrm{MHz}, \mathrm{CDCl}_{3}\right): \delta=7.82-8.02$ (m, $4 \mathrm{H}, \mathrm{H}-2 ", \mathrm{H}-6 "$ \& H-2', H-6'), 7.69 (d, 2H, J = 8.4 Hz, H-3", H-5"), 7.35 (d, 2H, J = 7.9 $\left.\mathrm{Hz}, \mathrm{H}-3^{\prime}, \mathrm{H}-5^{\prime}\right), 2.46$ (s, 3H, 4'-CH $)$ ppm; ${ }^{13} \mathrm{C}-\mathrm{NMR}\left(100 \mathrm{MHz}, \mathrm{CDCl}_{3}\right)$ : $\delta=164.9(\mathrm{C}, \mathrm{C}-2), 163.6(\mathrm{C}, \mathrm{C}-5), 142.5\left(\mathrm{C}, \mathrm{C}-4^{\prime}\right), 132.4\left(\mathrm{CH}, \mathrm{C}-3^{\prime \prime}\right.$, C-5"), 129.8 (CH, C-2', C-6'), 128.3 (CH, C-2", C-6"), 126.9 (CH, C-3', C-5'), 126.3 (C, C-1"), 122.9 (C, C-4"), 120.9 (C, C-1'), $21.7\left(\mathrm{CH}_{3}\right)$ ppm; FT-IR (KBr): transparent in the region of $(\mathrm{N}-\mathrm{H}$ str.) and $(\mathrm{C}=\mathrm{O}$ str. $)$, 1248 (C-O str.) $\mathrm{cm}^{-1}$; MS (ESI): $\mathrm{m} / \mathrm{z}=315.2(\mathrm{M}+1)^{+}, 317.1(\mathrm{M}+2)^{+}$in the ratio showing typical bromine isotope profile (1:1); Anal. Calcd. for $\mathrm{C}_{15} \mathrm{H}_{11} \mathrm{BrN}_{2} \mathrm{O}$ : C, 57.29; H, 3.50; N, 8.91. Found: C, 57.28; H, 3.48; $\mathrm{N}, 8.89$.

\section{5-(4"-Methoxyphenyl)-2-(4'-methylphenyl)-1,3,4-oxadiazole} (4k)

Yield 88.7 \%; m.p.: (Obs.) $148-149^{\circ} \mathrm{C}$, m.p.: (Lit.) $148-150{ }^{\circ} \mathrm{C}$; TLC $\mathrm{R}_{\mathrm{f}}=0.67$ [ethylacetate: hexane (3:7)]; ${ }^{1} \mathrm{H}-\mathrm{NMR}\left(400 \mathrm{MHz}, \mathrm{CDCl}_{3}\right): \delta$ 7.68-8.00 (m, 4H, H-2", H-6" \& H-2', H-6'), 7.40 (d, 2H, J = 8.0 Hz,

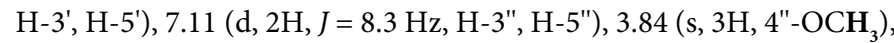
2.44 (s, 3H, 4'-CH $)$ ppm; ${ }^{13} \mathrm{C}-\mathrm{NMR}\left(100 \mathrm{MHz}, \mathrm{DMSO}-d_{6}\right): \delta 164.2(\mathrm{C}$, C-2), 162.8 (C, C-5), 159.7 (C, C-4"), 142.7 (C, C-4'), 129.7 (CH, C-2', C-6'), 128.5 (CH, C-2", C-6"), 127.3 (CH, C-3', C-5'), 121.2 (C, C-1'), 120.6 (C, C-1"), $114.5(\mathrm{CH}, \mathrm{C}-3 ", \mathrm{C}-5 "), 55.3\left(\mathrm{OCH}_{3}\right), 21.2\left(\mathrm{CH}_{3}\right) \mathrm{ppm}$; FT-IR (KBr): transparent in the region of (N-H str.) and (C=O str.), 1256 (C-O str.) $\mathrm{cm}^{-1}$; MS (ESI): $\mathrm{m} / \mathrm{z}=267.1(\mathrm{M}+1)^{+}$; Anal. Calcd. for $\mathrm{C}_{16} \mathrm{H}_{14} \mathrm{~N}_{2} \mathrm{O}_{2}: \mathrm{C}, 72.15 ; \mathrm{H}, 5.26 ; \mathrm{N}, 10.52$. Found: C, 72.14; H, 5.24; N, 10.49 .

\section{5-(4"-Methylphenyl)-2-(4'-methylphenyl)-1,3,4-oxadiazole} (41)

Yield 88 \%; m.p.: (Obs.) $179-180{ }^{\circ} \mathrm{C}$, m.p.: (Lit.) $179-180{ }^{\circ} \mathrm{C}$; TLC $\mathrm{R}_{\mathrm{f}}=0.73$ [ethylacetate: hexane (3:7)]; ${ }^{1} \mathrm{H}-\mathrm{NMR}\left(400 \mathrm{MHz}, \mathrm{DMSO}-d_{6}\right)$ : $\delta=7.74-7.95$ (m, 4H, H-2", H-6" \& H-2', H-6'), 7.38 (d, 2H, $J=8.4 \mathrm{~Hz}$, H-3', H-5'), 7.28 (d, 2H, J = 8.6 Hz, H-3", H-5"), 2.45 (s, 6H, 4', 4"-CH ppm; ${ }^{13} \mathrm{C}-\mathrm{NMR}\left(100 \mathrm{MHz}, \mathrm{DMSO}-d_{6}\right): \delta=166.8(\mathrm{C}, \mathrm{C}-2), 165.9(\mathrm{C}$, C-5), 144.7 (C, C-4"), 142.2 (C, C-4'), 130.1 (CH, C-2", C-6"), 129.8 (CH, C-2', C-6'), 127.2 (CH, C-3", C-5"), 127.0 (CH, C-3', C-5'), 120.3 (C, C-1"), $120.1\left(\mathrm{C}, \mathrm{C}-1^{\prime}\right), 21.7\left(\mathrm{CH}_{3}\right), 21.3\left(\mathrm{CH}_{3}\right)$ ppm; FT-IR $(\mathrm{KBr})$ : transparent in the region of (N-H str.) and ( $\mathrm{C}=\mathrm{O}$ str.), 1255 (C-O str.) $\mathrm{cm}^{-1}$; MS (ESI): $\mathrm{m} / \mathrm{z}=251.1(\mathrm{M}+1)^{+}$; Anal. Calcd. for $\mathrm{C}_{16} \mathrm{H}_{14} \mathrm{~N}_{2} \mathrm{O}: \mathrm{C}$, 76.77; H, 5.60; N, 11.19. Found: C, 76.76; H, 5.59; N, 11.18 .

\section{2-(4'-Methylphenyl)-5-(thien-2"-yl)-1,3,4-oxadiazole (4m)}

Yield 85 \%; m.p.: $126-127^{\circ} \mathrm{C}$; TLC $\mathrm{R}_{\mathrm{f}}=0.72$ [ethylacetate: hexane (3:7)]; ${ }^{1} \mathrm{H}-\mathrm{NMR}\left(400 \mathrm{MHz}, \mathrm{DMSO}-d_{6}\right): \delta=8.06(\mathrm{~d}, 2 \mathrm{H}, J=8.1 \mathrm{~Hz}, \mathrm{H}-2$, 
Citation: Kumar V, Kumar M, Beniwal V, Gupta GK, Kumar S, et al. (2016) Synthesis of Some Aroylhydrazones and 2,5-Disubstituted-1,3,4Oxadiazoles as DNA Photocleaving Agents. Med chem (Los Angeles) 6: 474-485. doi:10.4172/2161-0444.1000386

H-6'), 7.28-7.98 (m, 5H, H-3", H-4", H-5" \& H-3', H-5'), 2.42 (s, 3H, 4'$\left.\mathrm{CH}_{3}\right) \mathrm{ppm} ;{ }^{13} \mathrm{C}-\mathrm{NMR}\left(100 \mathrm{MHz}, \mathrm{DMSO}-d_{6}\right): \delta=165.9(\mathrm{C}, \mathrm{C}-2), 164.4$ (C, C-5), 142.3 (C, C-4'), 132.0 (CH, C-5"), $130.0\left(\mathrm{CH}, \mathrm{C}-2^{\prime}, \mathrm{C}-6^{\prime}\right), 129.5$ (CH, C-3"), $128.6(\mathrm{CH}, \mathrm{C}-4 "), 126.9$ (C, C-2"), 126.8 (CH, C-3', C-5'), $120.6\left(\mathrm{C}, \mathrm{C}-1^{\prime}\right), 21.3\left(\mathrm{CH}_{3}\right)$ ppm; FT-IR $(\mathrm{KBr})$ : transparent in the region of ( $\mathrm{N}-\mathrm{H}$ str.) and (C=O str.), 1249 (C-O str.) $\mathrm{cm}^{-1}$; MS (ESI): $\mathrm{m} / \mathrm{z}=$ $243.3(\mathrm{M}+1)+$; Anal. Calcd. for $\mathrm{C}_{13} \mathrm{H}_{10} \mathrm{~N}_{2} \mathrm{OS}$ : C, 64.38; H, 4.13; N, 11.55 . Found: $\mathrm{C}, 64.36 ; \mathrm{H}, 4.10 ; \mathrm{N}, 11.54$.

\section{5-(Fur-2"-yl)-2-(4'-methylphenyl)-1,3,4-oxadiazole (4n)}

Yield $84.9 \%$; m.p.: (Obs.) $131-132{ }^{\circ} \mathrm{C}$, m.p.: (Lit.) 131-132 ${ }^{\circ} \mathrm{C}$; TLC $\mathrm{R}_{\mathrm{f}}=0.60$ [ethylacetate: hexane (3:7)]; ${ }^{1} \mathrm{H}-\mathrm{NMR}\left(400 \mathrm{MHz}, \mathrm{DMSO}-d_{6}\right)$ : $\delta=7.95$ (d, $\left.2 \mathrm{H}, J=8.0 \mathrm{~Hz}, \mathrm{H}-2^{\prime}, \mathrm{H}-6^{\prime}\right), 7.21-7.85$ (m, 5H, H-3", H-4", $\mathrm{H}-5 "$ \& H-3', H-5'), 2.44 (s, 3H, 4'-CH $)$ ppm; ${ }^{13} \mathrm{C}-\mathrm{NMR}(100 \mathrm{MHz}$, DMSO- $\left.d_{6}\right): \delta=165.4$ (C, C-2), 164.3 (C, C-5), 142.0 (C, C-4'), 133.5 (CH, C-5"), 129.9 (CH, C-2', C-6'), 128.6 (C, C-2"), 127.0 (CH, C-3', C-5'), 120.9 (C, C-1'), 115.2 (CH, C-3"), $113.8(\mathrm{CH}, \mathrm{C}-4 "), 21.3\left(\mathrm{CH}_{3}\right)$ ppm; FT-IR (KBr): transparent in the region of $(\mathrm{N}-\mathrm{H}$ str. $)$ and $(\mathrm{C}=\mathrm{O}$ str.), 1251 (C-O str.) $\mathrm{cm}^{-1}$; MS (ESI): $\mathrm{m} / \mathrm{z}=227.1(\mathrm{M}+1)^{+}$; Anal. Calcd. for $\mathrm{C}_{13} \mathrm{H}_{10} \mathrm{~N}_{2} \mathrm{O}_{2}$ : C, 69.00; H, 4.42; N, 12.38. Found: C, 69.00; H, 4.40; $\mathrm{N}, 12.37$.

\section{5-(1",3"-Diphenyl-4"-pyrazolyl)-2-(4'-nitrophenyl)-1,3,4- oxadiazole (40)}

Yield $86.4 \%$; m.p.: $210-211^{\circ} \mathrm{C}$; TLC $\mathrm{R}_{\mathrm{f}}=0.60$ [ethylacetate: hexane (3:7)]; ${ }^{1} \mathrm{H}-\mathrm{NMR}$ (400 MHz, DMSO- $\left.d_{6}\right): \delta=9.38$ (s, $\left.1 \mathrm{H}, \mathrm{H}-5 "\right), 8.26-8.44$ (m, 4H, H-Ph'), 7.42-8.03 (m, 10H, H-Ph"' \& H-Ph"') ppm; ${ }^{13} \mathrm{C}-\mathrm{NMR}$ $\left(100 \mathrm{MHz}, \mathrm{DMSO}-d_{6}\right): \delta=164.1$ (C, C-5), 161.6 (C, C-2), 150.8 (C, C-3"), 149.0 (C, C-4'), 138.6 (C, C-1"'), 131.4 (C, C-1"'"), $131.1(\mathrm{CH}$, C-5"), 129.4 (CH, C-2', C-6'), 128.9 (CH, C-3"'", C-5"'), 128.8 (CH, C-3"', C-5"'), 128.7 (CH, C-4"'), 128.4 (CH, C-2"'", C-6"'), 127.9 (CH, C-4"'), 127.2 (C, C-1'), 124.3 (CH, C-3', C-5'), 118.9 (CH, C-2"', C-6"'), 105.5 (C, C-4") ppm; FT-IR (KBr): transparent in the region of (N-H str.) and (C=O str.), 1256 (C-O str.) $\mathrm{cm}^{-1}$; MS (ESI): $\mathrm{m} / \mathrm{z}=410.1(\mathrm{M}+1)^{+}$; Anal. Calcd. for $\mathrm{C}_{23} \mathrm{H}_{15} \mathrm{~N}_{5} \mathrm{O}_{3}: \mathrm{C}, 67.46 ; \mathrm{H}, 3.67 ; \mathrm{N}, 17.11$. Found: C, 67.44; $\mathrm{H}$, $3.65 ; \mathrm{N}, 17.10$.

\section{2-(4'-Nitrophenyl)-5-(4"-nitrophenyl)-1,3,4-oxadiazole (4p)}

Yield 89 \%; m.p.: (Obs.) $127-128^{\circ} \mathrm{C}$, m.p.: (Lit.) $127-129^{\circ} \mathrm{C}$; TLC $\mathrm{R}_{\mathrm{f}}=0.68$ [ethylacetate: hexane (3:7)]; ${ }^{1} \mathrm{H}-\mathrm{NMR}\left(400 \mathrm{MHz}, \mathrm{DMSO}-d_{6}\right)$ : $\delta=8.32-8.54\left(\mathrm{~m}, 8 \mathrm{H}, \mathrm{H}-\mathrm{Ph}^{\prime} \& \mathrm{H}-\mathrm{Ph} "\right) \mathrm{ppm} ;{ }^{13} \mathrm{C}-\mathrm{NMR}(100 \mathrm{MHz}$, DMSO- $\left.d_{6}\right): \delta=163.1$ (C, C-2), 162.6 (C, C-5), 149.3 (C, C-4'), 149.1 (C, C-4"), 130.1 (C, C-1"), 129.3 (C, C-1'), 127.2 (CH, C-2', C-6'), 126.9 (CH, C-2", C-6"), 124.6 (CH, C-3', C-5'), 124.4 (CH, C-3", C-5") ppm; FT-IR (KBr): transparent in the region of $(\mathrm{N}-\mathrm{H}$ str.) and $(\mathrm{C}=\mathrm{O}$ str.), 1546 ( $\mathrm{NO}_{2}$ asymmetric str.), 1351 ( $\mathrm{NO}_{2}$ symmetric str.), 1257 (C-O str.) $\mathrm{cm}^{-1}$; MS (ESI): $\mathrm{m} / \mathrm{z}=313.0(\mathrm{M}+1)^{+}$; Anal. Calcd. for $\mathrm{C}_{14} \mathrm{H}_{8} \mathrm{~N}_{4} \mathrm{O}_{5}: \mathrm{C}$, 53.85; H, 2.56; N, 17.95. Found: C, 53.83; H, 2.54; N, 17.93 .

5-(4"-Bromophenyl)-2-(4'-nitrophenyl)-1,3,4-oxadiazole (4q)

Yield $85 \%$; m.p.: $197-198{ }^{\circ} \mathrm{C}$; TLC $\mathrm{R}_{\mathrm{f}}=0.74$ [ethylacetate: hexane (3:7)]; ${ }^{1} \mathrm{H}-\mathrm{NMR}\left(400 \mathrm{MHz}, \mathrm{DMSO}-d_{6}\right): \delta=8.39-8.46(\mathrm{~m}, 4 \mathrm{H}, \mathrm{H}-\mathrm{Ph})$, 7.63-7.81 (m, 4H, H-Ph") ppm; ${ }^{13} \mathrm{C}-\mathrm{NMR}\left(100 \mathrm{MHz}, \mathrm{DMSO}-d_{6}\right): \delta=$ 163.6 (C, C-5), 163.2 (C, C-2), 148.8 (C, C-4'), 132.3 (CH, C-3", C-5"), 129.8 (C, C-1'), 128.4 (CH, C-2", C-6"), 127.3 (CH, C-2', C-6'), 126.3 (C, C-1"), 124.3 (CH, C-3', C-5'), 122.9 (C, C-4") ppm; FT-IR (KBr): transparent in the region of (N-H str.) and (C=O str.), 1250 (C-O str.) $\mathrm{cm}^{-1}$; MS (ESI): $\mathrm{m} / \mathrm{z}=346.1(\mathrm{M}+1)^{+}, 348.1(\mathrm{M}+2)^{+}$in the ratio showing typical bromine isotope profile (1:1); Anal. Calcd. for $\mathrm{C}_{14} \mathrm{H}_{8} \mathrm{BrN}_{3} \mathrm{O}_{3}$ : C,
48.68; H, 2.32; N, 12.17. Found: C, 48.66; H, 2.30; N, 12.14.

\section{5-(4"-Methoxyphenyl)-2-(4'-nitrophenyl)-1,3,4-oxadiazole} (4r)

Yield 90 \%; m.p.: $176-177^{\circ} \mathrm{C}$; TLC $\mathrm{R}_{\mathrm{f}}=0.66$ [ethylacetate: hexane (3:7)]; ${ }^{1} \mathrm{H}-\mathrm{NMR}\left(400 \mathrm{MHz}, \mathrm{DMSO}-d_{6}\right): \delta=8.37-8.46\left(\mathrm{~m}, 4 \mathrm{H}, \mathrm{H}-\mathrm{Ph}^{\prime}\right)$, $8.10\left(\mathrm{~d}, 2 \mathrm{H}, J=8.4 \mathrm{~Hz}, \mathrm{H}-2^{\prime \prime}, \mathrm{H}-6 "\right), 7.15$ (d, $2 \mathrm{H}, J=8.2 \mathrm{~Hz}, \mathrm{H}-3^{\prime \prime}$, H-5"), 3.90 (s, 3H, 4"-OCH $)$ ppm; ${ }^{13} \mathrm{C}-\mathrm{NMR}\left(100 \mathrm{MHz}, \mathrm{DMSO}-d_{6}\right): \delta$ $=162.3(\mathrm{C}, \mathrm{C}-5), 162.1$ (C, C-2), 159.6 (C, C-4"), 149.1 (C, C-4'), 129.1 (C, C-1'), $128.6\left(\mathrm{CH}, \mathrm{C}-2^{\prime \prime}, \mathrm{C}-6 "\right), 127.6\left(\mathrm{CH}, \mathrm{C}-2^{\prime}, \mathrm{C}-6^{\prime}\right), 124.3(\mathrm{CH}$, C-3', C-5'), 120.9 (C, C-1"), 114.6 (CH, C-3", C-5"), $55.3\left(\mathrm{OCH}_{3}\right)$ ppm; FT-IR (KBr): transparent in the region of $(\mathrm{N}-\mathrm{H}$ str.) and ( $\mathrm{C}=\mathrm{O}$ str.), 1248 (C-O str.) $\mathrm{cm}^{-1}$; MS (ESI): $\mathrm{m} / \mathrm{z}=298.2(\mathrm{M}+1)^{+}$; Anal. Calcd. for $\mathrm{C}_{15} \mathrm{H}_{11} \mathrm{~N}_{3} \mathrm{O}_{4}: \mathrm{C}, 60.56 ; \mathrm{H}, 3.70 ; \mathrm{N}, 14.13$. Found: $\mathrm{C}, 60.54 ; \mathrm{H}, 3.69 ; \mathrm{N}$, 14.11 .

\section{5-(4"-Methylphenyl)-2-(4'-nitrophenyl)-1,3,4-oxadiazole} (4s)

Yield 87.9 \%; m.p.: $188-189^{\circ} \mathrm{C}$; TLC $\mathrm{R}_{\mathrm{f}}=0.70$ [ethylacetate: hexane (3:7)]; ${ }^{1} \mathrm{H}-\mathrm{NMR}\left(400 \mathrm{MHz}, \mathrm{CDCl}_{3}\right): \delta=8.31-8.42\left(\mathrm{~m}, 4 \mathrm{H}, \mathrm{H}-\mathrm{Ph}^{\prime}\right), 8.05$ (d, 2H, $J=8.4 \mathrm{~Hz}, \mathrm{H}-2 ", \mathrm{H}-6 "), 7.37$ (d, 2H, $J=8.2 \mathrm{~Hz}, \mathrm{H}-3 ", \mathrm{H}-5 ")$, 2.46 (s, 3H, 4"-CH $)$ ppm; ${ }^{13} \mathrm{C}-\mathrm{NMR}\left(100 \mathrm{MHz}, \mathrm{CDCl}_{3}\right): \delta=165.7(\mathrm{C}$ C-5), 162.6 (C, C-2), 149.5 (C, C-4'), 143.1 (C, C-4"), 130.0 (CH, C-2", C-6"), 129.0 (C, C-1'), 127.7 (CH, C-2', C-6'), 127.1 (CH, C-3", C-5"), $124.4\left(\mathrm{CH}, \mathrm{C}-3^{\prime}, \mathrm{C}-5^{\prime}\right), 120.5$ (C, C-1"), $21.8\left(\mathrm{CH}_{3}\right)$ ppm; FT-IR (KBr): transparent in the region of ( $\mathrm{N}-\mathrm{H}$ str.) and ( $\mathrm{C}=\mathrm{O}$ str.), 1254 (C-O str.) $\mathrm{cm}^{-1}$; MS (ESI): $\mathrm{m} / \mathrm{z}=282.3(\mathrm{M}+1)^{+}$; Anal. Calcd. for $\mathrm{C}_{15} \mathrm{H}_{11} \mathrm{~N}_{3} \mathrm{O}_{3}: \mathrm{C}$, 63.99; H, 3.91; N, 14.93. Found: C, 63.97; H, 3.89; N, 14.91 .

\section{2-(4'-Nitrophenyl)-5-(thien-2"-yl)-1,3,4-oxadiazole (4t)}

Yield 84.9 \%; m.p.: $165-166{ }^{\circ} \mathrm{C}$; TLC $\mathrm{R}_{\mathrm{f}}=0.57$ [ethylacetate: hexane (3:7)]; ${ }^{1} \mathrm{H}-\mathrm{NMR}\left(400 \mathrm{MHz}, \mathrm{DMSO}-d_{6}\right): \delta=8.35-8.46(\mathrm{~m}, 4 \mathrm{H}$, H-Ph'), 7.29-7.96 (m, 3H, H-3", H-4", H-5") ppm; ${ }^{13} \mathrm{C}-\mathrm{NMR}(100 \mathrm{MHz}$, DMSO- $\left.d_{6}\right): \delta=164.3$ (C, C-5), 162.4 (C, C-2), 148.9 (C, C-4'), 133.5 (CH, C-5"), $131.6(\mathrm{CH}, \mathrm{C}-3 "), 130.6(\mathrm{CH}, \mathrm{C}-4 "), 128.7$ (C, C-1'), 128.4 (C, C-2"), 127.7 (CH, C-2', C-6'), 124.3 (CH, C-3', C-5') ppm; FT-IR $(\mathrm{KBr})$ : transparent in the region of $(\mathrm{N}-\mathrm{H}$ str. $)$ and $(\mathrm{C}=\mathrm{O}$ str. $), 1248$ (C-O str.) $\mathrm{cm}^{-1}$; MS (ESI): $\mathrm{m} / \mathrm{z}=274.0(\mathrm{M}+1)^{+}$; Anal. Calcd. for $\mathrm{C}_{12} \mathrm{H}_{7}$ $\mathrm{N}_{3} \mathrm{O}_{3} \mathrm{~S}: \mathrm{C}, 52.75 ; \mathrm{H}, 2.56 ; \mathrm{N}, 15.38$. Found: C, 52.73; H, 2.54; N, 15.36.

\section{5-(Fur-2"-yl)-2-(4'-nitrophenyl)-1,3,4-oxadiazole (4u)}

Yield $85.5 \%$; m.p.: $145-146{ }^{\circ} \mathrm{C}$; TLC $\mathrm{R}_{\mathrm{f}}=0.55$ [ethylacetate: hexane (3:7)]; ${ }^{1} \mathrm{H}-\mathrm{NMR}$ (400 MHz, DMSO- $\left.d_{6}\right): \delta=8.31-8.49(\mathrm{~m}, 4 \mathrm{H}$, H-Ph'), 7.01-7.85 (m, 3H, H-3", H-4", H-5") ppm; ${ }^{13} \mathrm{C}-\mathrm{NMR}(100 \mathrm{MHz}$, DMSO- $\left.d_{6}\right): \delta=164.5$ (C, C-5), 163.0 (C, C-2), 149.4 (C, C-4'), 133.8 (CH, C-5"), 129.7 (C, C-2"), 129.0 (C, C-1'), 127.8 (CH, C-2', C-6'), $124.4\left(\mathrm{CH}, \mathrm{C}-3^{\prime}, \mathrm{C}-5^{\prime}\right), 114.6\left(\mathrm{CH}, \mathrm{C}-3^{\prime \prime}\right), 114.0(\mathrm{CH}, \mathrm{C}-4 ")$ ppm; FTIR $(\mathrm{KBr})$ : transparent in the region of (N-H str.) and ( $\mathrm{C}=\mathrm{O}$ str.), 1249 (C-O str.) $\mathrm{cm}^{-1}$; MS (ESI): $\mathrm{m} / \mathrm{z}=258.1(\mathrm{M}+1)^{+}$; Anal. Calcd. for $\mathrm{C}_{12} \mathrm{H}_{7}$ $\mathrm{N}_{3} \mathrm{O}_{4}: \mathrm{C}, 56.01 ; \mathrm{H}, 2.72 ; \mathrm{N}, 16.34$. Found: $\mathrm{C}, 56.00 ; \mathrm{H}, 2.71 ; \mathrm{N}, 16.32$.

\section{Biological Activity}

\section{DNA photocleavage study}

DNA photocleavage experiment was performed by taking $10 \mu \mathrm{L}$ solution containing plasmid DNA in TE (Tris $10 \mathrm{mM}$, EDTA $0.01 \mathrm{mM}$, $\mathrm{pH} 8.0$ ) buffer in the presence of $40 \mu \mathrm{g}$ of synthesized compounds. The sample solution held in caps of polyethylene microcentrifuge tubes were placed directly on the surface of a trans-illuminator $(8000 \mathrm{~mW} /$ $\mathrm{cm})$ at $360 \mathrm{~nm}$ and were irradiated for $30 \mathrm{~min}$ at room temperature. 
Citation: Kumar V, Kumar M, Beniwal V, Gupta GK, Kumar S, et al. (2016) Synthesis of Some Aroylhydrazones and 2,5-Disubstituted-1,3,4Oxadiazoles as DNA Photocleaving Agents. Med chem (Los Angeles) 6: 474-485. doi:10.4172/2161-0444.1000386

After irradiation, samples were further incubated at $37^{\circ} \mathrm{C}$ for $1 \mathrm{~h}$. Irradiated samples were mixed with $6 \mathrm{X}$ loading dye containing $0.25 \%$ bromophenol blue and $30 \%$ glycerol. The samples were then analyzed by electrophoresis on a $0.8 \%$ agarose horizontal slab gel in Tris-acetate EDTA buffer (40 mM Tris, $20 \mathrm{mM}$ acetic acid, 1 mM EDTA, pH: 8.0). Untreated plasmid DNA was maintained as a control in each run of gel electrophoresis which was carried out at $5 \mathrm{~V} / \mathrm{cm}$ for $2.0 \mathrm{~h}$. Gel was stained with ethidium bromide $(1 \mu \mathrm{g} / \mathrm{mL})$ and photographed under UV light [37]. To account the effect of synthesized compounds on DNA, the band intensities were analyzed using the MYImage Analysis software provided by Thermo Fisher Scientific Inc.

\section{Results and Discussion}

\section{Chemistry}

A variety of disubstituted-1,3,4-oxadiazoles had already been synthesized using different toxic reagents like phosphorus oxychloride [38], phosphorus pentaoxide [39] and acetic anhydride [40]. In the past few years, organic synthesis acquired various advantages such as shorter reaction time, higher regio-selectivity [41] and use of greener solvents or reagents with low toxicity profile. In this concern, organoiodine(III) reagents are well known for their non-toxic and eco-friendly behavior in organic synthesis $[42,43]$. Due to low toxicity and selective nature [44], they have also been extensively used for the synthesis of various heterocycles. In continuation of our interest to synthesize biological active azole derivatives, herein, we report the synthesis of some new 2,5-disubstituted-1,3,4-oxadiazoles by oxidative transformation of various newly synthesized hydrazones by iodobenzene diacetate (IBD), a hypervalent iodine (III) reagent in dichloromethane under mild reaction conditions.

The substituted aroylhydrazones $\mathbf{3}$ were obtained by the condensation of aroylhydrazines 1 with 3-aryl-1-phenyl- $1 H$-pyrazole4-carbaldehydes/benzaldehyde derivatives 2 in a mixture of ethanol and dichloromethane in presence of catalytic amount of concentrated sulfuric acid under reflux according to a literature method which was used to synthesize some different derivatives $[45,46]$. The final products 4 were obtained in $84-91 \%$ yields with high purity via oxidative cyclization of 3 by treating with 1.1 equivalent of IBD under mild conditions (Scheme 1). Although some 1,3,4-oxadiazole derivatives had been prepared via oxidation of a few substituted hydrazones such as $N$-acyl hydrazones [47] with 1.1 equivalent of iodobenzene diacetate in dichloromethane (DCM) at room temperature, in the present investigation total 42 compounds were prepared. The compounds were characterized on the basis of their FT-IR, ${ }^{1} \mathrm{H},{ }^{13} \mathrm{C}$ NMR and mass spectral data. The FT-IR spectra of compounds 3a-u showed absorption bands for $-\mathrm{NH}$ and $-\mathrm{C}=\mathrm{O}$ stretching vibrations at 3427 and $1668 \mathrm{~cm}^{-1}$, respectively. In ${ }^{1} \mathrm{H}$ NMR spectra, the compounds $\mathbf{3 a}$, $3 \mathbf{h}$ and 30 displayed two characteristic signals due to 5 '- $\mathrm{H}$ of pyrazole ring and $\mathrm{N}=\mathrm{CH}$ at $\delta 8.96$ and 8.61 , respectively. A characteristic downfield signal at $\delta 11.76$ was appeared due to the $-\mathrm{NH}$ proton and rest of the protons exhibited multiplet in the aromatic region. The chemical shifts in ${ }^{13} \mathrm{C}$ NMR spectra of $\mathbf{3 a}, \mathbf{3 h}$ and $\mathbf{3 o}$ (considering as representative cases) at $\delta 140.1-144.1,126.6-127.5$ and $160.9-163.4$ correspond to $\mathrm{N}=\mathrm{CH}$, pyrazole-5' and carbonyl carbon atoms, respectively.

The structures of final products $4 \mathbf{a}-\mathbf{u}$ were established by comparing FT-IR, ${ }^{1} \mathrm{H}$ and ${ }^{13} \mathrm{C}$ NMR spectral data with those of the compounds $\mathbf{3 a}-$ u. The FT-IR spectra of 4 were found transparent in region of $-\mathrm{NH}$ and $-\mathrm{C}=\mathrm{O}$ stretching and thus confirmed the successful oxidation of $\mathbf{3}$ into 4. Disappearance of chemical shifts at $\delta 8.38-8.72(\mathrm{~N}=\mathrm{CH})$ and 11.70$12.14(-N H)$ in ${ }^{1} \mathrm{H}$ NMR spectra of the products $(\mathbf{4 a}-\mathbf{u})$ confirmed the successful conversion of aroylhydrazones into 2,5-disubstituted-1,3,4oxadiazoles. The ${ }^{13} \mathrm{C}$ NMR spectra displayed signals at around $\delta 159.9$, 164.0 for two oxadiazole carbons, however, signals at $\delta 152.0,106.5$ and 131.6 were appeared due to pyrazole ring carbons $3 ", 4 "$ and $5 "$, respectively. In ${ }^{13} \mathrm{C}$ NMR spectrum, disappearance of a characteristic signal in range of $\delta 140.1-144.1$ due to $\mathrm{N}=\mathrm{CH}$ functionality further confirmed the formation of oxadiazoles. The physical data of all the synthesized compounds 4 is given in the Table 1 .

The probable mechanism involved in synthesis of oxadiazole is given in Figure 1. The intermediate $\mathbf{I}$ is formed by the attack of hydrazone nitrogen on iodine of iodobenzene diacetate followed by elimination of acetate ion. The rate of reaction may depend upon the electron density on the carbon-a and carbon-b. Higher electro-positive character on carbon-b accelerates the attacking tendency of oxygen and the rate of reaction. Whereas, increased in positive charge on carbon-a reduces the attacking tendency of oxygen and hence retards the rate of reaction. On this basis, it is assumed that the presence of electronwithdrawing group at para position of phenyl ring $\left(\mathrm{R}^{2}\right)$ facilitates the rate of reaction. On the other hand, electron-releasing group at para

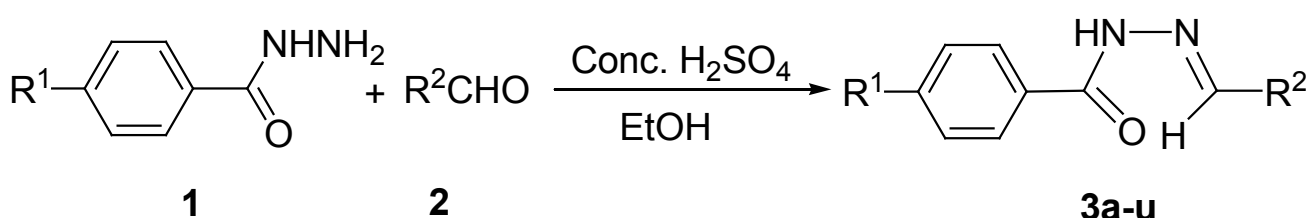

$$
\begin{aligned}
& \mathrm{R}^{1}=\mathrm{H}, \mathrm{CH}_{3}, \mathrm{NO}_{2} \\
& \mathrm{R}^{2}=\text { aryl, heteroaryl }
\end{aligned}
$$<smiles>[R4]c1ccc(-c2nn(CC(C)C)c([R])[n+]2C)cc1</smiles>

Scheme 1: Synthesis of substituted aroylhydrazones (3a-u) and oxadiazoles (4a-u). 
Citation: Kumar V, Kumar M, Beniwal V, Gupta GK, Kumar S, et al. (2016) Synthesis of Some Aroylhydrazones and 2,5-Disubstituted-1,3,4Oxadiazoles as DNA Photocleaving Agents. Med chem (Los Angeles) 6: 474-485. doi:10.4172/2161-0444.1000386

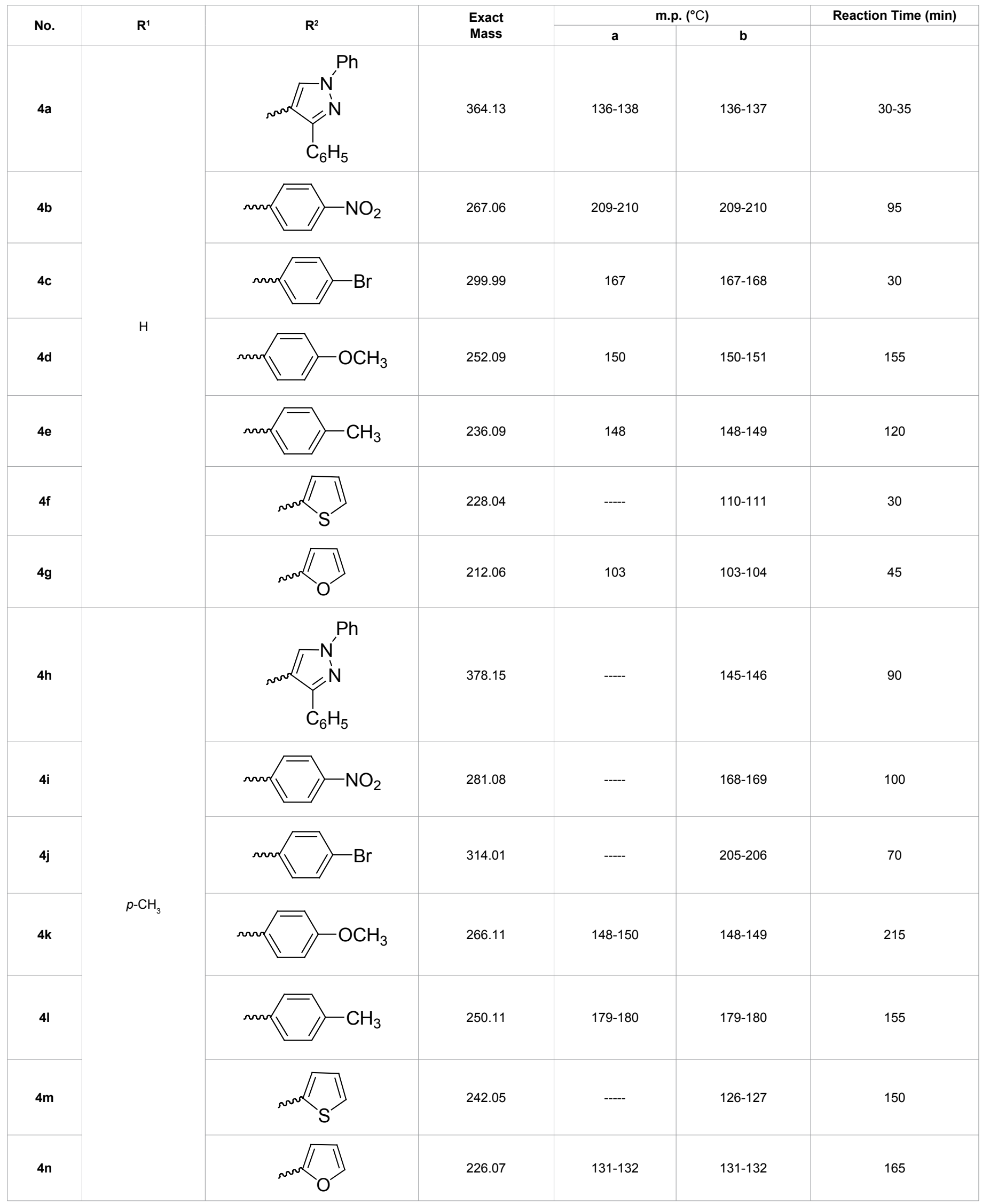




\begin{tabular}{|c|c|c|c|c|c|c|}
\hline 40 & & $\sqrt{L}^{\mathrm{Ph}} \mathrm{N}^{\mathrm{N}}$ & 409.12 & ---- & $210-211$ & 70 \\
\hline $4 p$ & & $-\mathrm{NO}_{2}$ & 312.05 & $127-129$ & $127-128$ & 90 \\
\hline $4 q$ & $p-\mathrm{NO}_{2}$ & & 344.97 & ---- & $197-198$ & 30 \\
\hline $4 r$ & & $\operatorname{sun}$ & 297.07 & ---- & 176-177 & 190 \\
\hline $4 s$ & & $\mathrm{CH}_{3}$ & 281.08 & --- & $188-189$ & 130 \\
\hline $4 t$ & & & 273.02 & ---- & 165-166 & 70 \\
\hline $4 u$ & & & 257.04 & ---- & $145-146$ & 40 \\
\hline
\end{tabular}

aLiterature m.p.; ${ }^{\mathrm{b} O b s e r v e d ~ m . p . ~}$

Table 1: Physical data of oxadiazoles (4).

position of phenyl ring $\left(\mathrm{R}^{2}\right)$ decreases rate of reaction by increasing the electron density at carbon-b.

On the other side, it is assumed that electron-withdrawing group attached to para position of phenyl ring $\left(\mathrm{R}^{1}\right)$ decreases the electron density on carbon-a and thus facilitates the elimination of proton attached to nitrogen but retains the attacking tendency of oxygen to carbon-b. While electron-releasing group attached to para position at phenyl ring decreases the electro-positive character of carbon-a and rate of elimination of proton as a result the rate of reaction gets decreased. Thus, phenyl ring having electron-releasing group attached to either carbon-a or b decreases the rate of reaction (Figure 2).

\section{Biological Evaluation}

\section{Plasmid DNA photocleavage study}

The DNA photocleavage study was performed using agarose gel electrophoresis method and results are presented in Figures 3 and 4. There was a significant decrease in intensity of DNA band in case of aroylhydrazones and oxadiazoles as compared to the control DNA.

In case of substituted aroylhydrazones $\mathbf{3 a - c}, \mathbf{3 h}, \mathbf{3 j}$, 3o-s (lane 2-4, 9, 11 and 17-21), decreased intensity of plasmid DNA as compared to control (lane 1) indicated the cleavage of DNA forms. In lane 11, 17-21 the compounds $\mathbf{3 j}$ and 3o-s were responsible for a complete fragmentation of supercoiled (Form I) into open circular (Form II) and linear (Form III) DNA. The intensity of Form III (linear form) was found to be increased in case of compounds $\mathbf{3} \mathbf{a}-\mathbf{c}, \mathbf{3} \mathbf{h}, \mathbf{3} \mathbf{j}$ (lane 2-4, 9 and 11) while Form I (supercoiled DNA) was decreased. Moreover, an appearance of the Form III in between Form I and II was observed due to nicking of super coiled DNA. Aroylhydrazones were found to be more active cleaving agent causing fragmentation of plasmid DNA into linear (Form III) DNA.
Unsymmetrical oxadiazoles derivatives (4) also exhibited efficient DNA cleavage property as presented in Figure 4 . On irradiation with UV light, the compounds $\mathbf{4 a - b}, \mathbf{4 d - h}, \mathbf{4 s}$ (lane 2-3, 5-9 and 21) were found to show cleavage of supercoiled (Form I) into open circular (Form II) DNA. In lane 12 , the compound $4 \mathbf{k}$ containing methoxy group at para-position of phenyl ring $\left(\mathrm{R}^{2}\right)$ was found to be the most effective cleaving agent which was particularly responsible for the complete fragmentation of plasmid DNA. It was found that compounds bearing electron-releasing substituent at para-position of phenyl ring $\left(\mathrm{R}^{2}\right)$ increase the cleavage potential of oxadiazole compounds. Moreover, higher intensity of the Form II (open circular DNA) was observed in case of aroylhydrazones containing pyrazole moiety. Furthermore, para substitution on phenyl ring also increases the cleavage action of the aroylhydrazone compounds on the plasmid DNA.

\section{Conclusion}

In the present investigation, we reported the synthesis of total 21 unsymmetrical 1,3,4-oxadiazole derivatives via oxidative cyclization of their corresponding 21 aroylhydrazones using IBD under solvent conditions and thus explored potential utility of organoiodine(III) reagents on a variety of hydrazone derivatives bearing different electron-withdrawing as well as electron-donating group substituents. It has been observed that phenyl ring $\left(\mathrm{R}^{1}\right)$ having electron-releasing group at para-position attached to carbon-a or b decreases the rate of reaction. Structures of the synthesized compounds were established on the basis of results obtained from their NMR spectral data. In DNA photocleavage study, it has been observed that compounds 3a-u and 4a-u have shown moderate DNA photocleavage activity. However, 3a$\mathbf{u}$, in particular, exhibited more photocleavage potential as compared to $4 \mathbf{a}-\mathbf{u}$. 
Citation: Kumar V, Kumar M, Beniwal V, Gupta GK, Kumar S, et al. (2016) Synthesis of Some Aroylhydrazones and 2,5-Disubstituted-1,3,4Oxadiazoles as DNA Photocleaving Agents. Med chem (Los Angeles) 6: 474-485. doi:10.4172/2161-0444.1000386

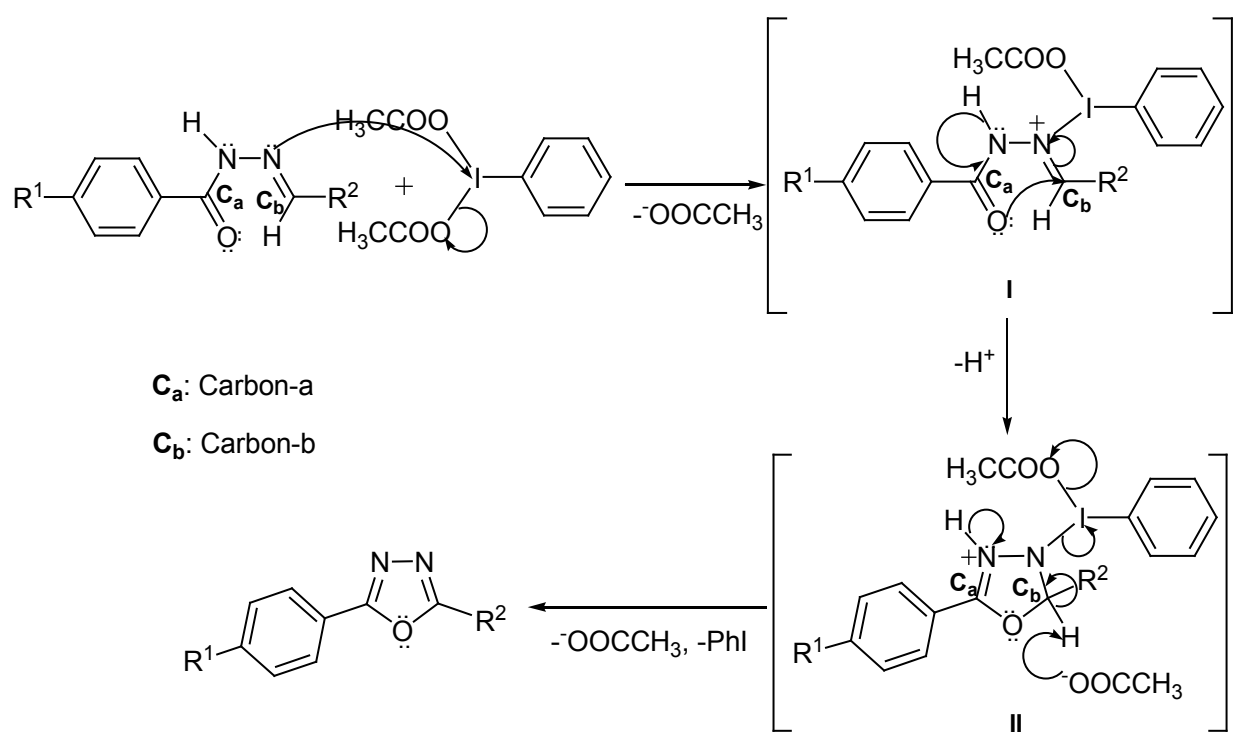

Figure 1: Proposed mechanism for the synthesis of oxadiazoles 4.

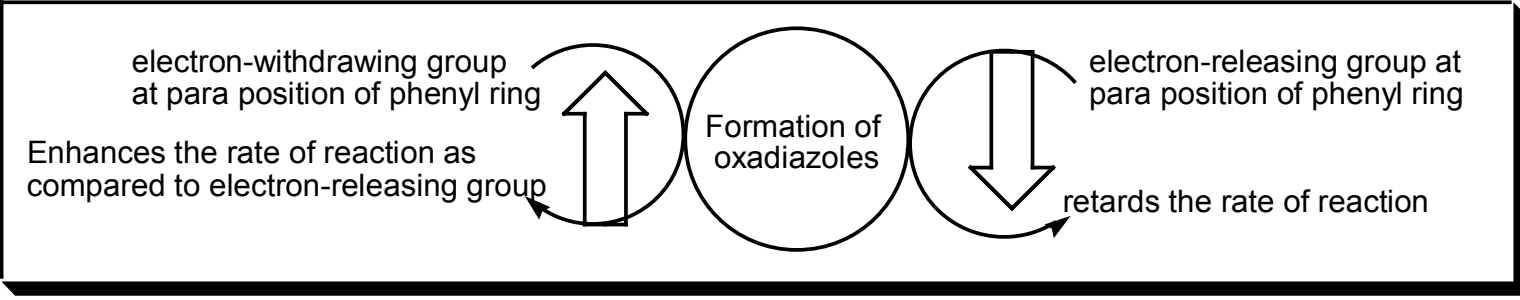

Figure 2: Effect of substitution on the formation of oxadiazoles.
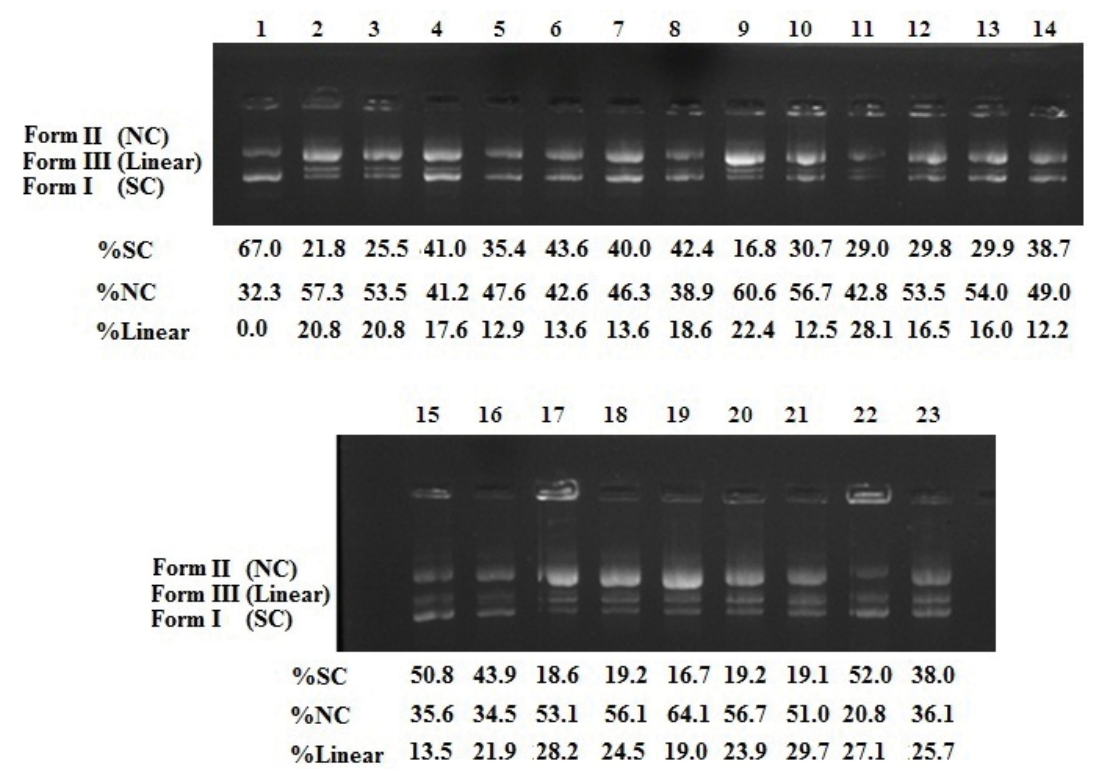

Figure 3: Plasmid DNA photocleavage of hydrazones 3a-u. Lane 1: Control plasmid DNA+UV+DMSO, Lane 2: DNA+40 $\mu \mathrm{g}$ 3a, Lane 3: DNA+40 $\mu \mathrm{g}$ 3b, Lane 4: DNA+40 $\mu \mathrm{g}$ 3c, Lane 5: DNA+40 $\mu \mathrm{g}$ 3d, Lane 6: DNA+40 $\mu \mathrm{g}$ 3e, Lane 7: DNA+40 $\mu \mathrm{g}$ 3f, Lane 8: DNA+40 $\mu \mathrm{g}$ 3g, Lane 9: DNA+40 $\mu \mathrm{g}$ 3h, Lane 10 : DNA+40 $\mu \mathrm{g}$ 3i

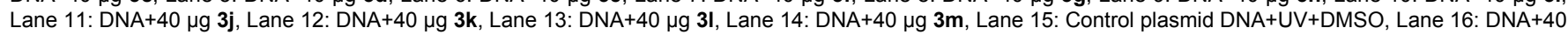

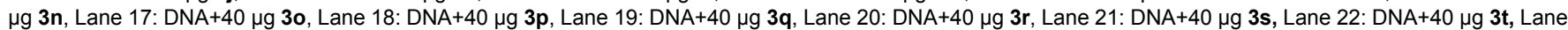
23: $\mathrm{DNA}+40 \mu \mathrm{g} 3 \mathrm{u}$, respectively. Values under each lane represent percentage contribution of Form I and II. 
Citation: Kumar V, Kumar M, Beniwal V, Gupta GK, Kumar S, et al. (2016) Synthesis of Some Aroylhydrazones and 2,5-Disubstituted-1,3,4Oxadiazoles as DNA Photocleaving Agents. Med chem (Los Angeles) 6: 474-485. doi:10.4172/2161-0444.1000386
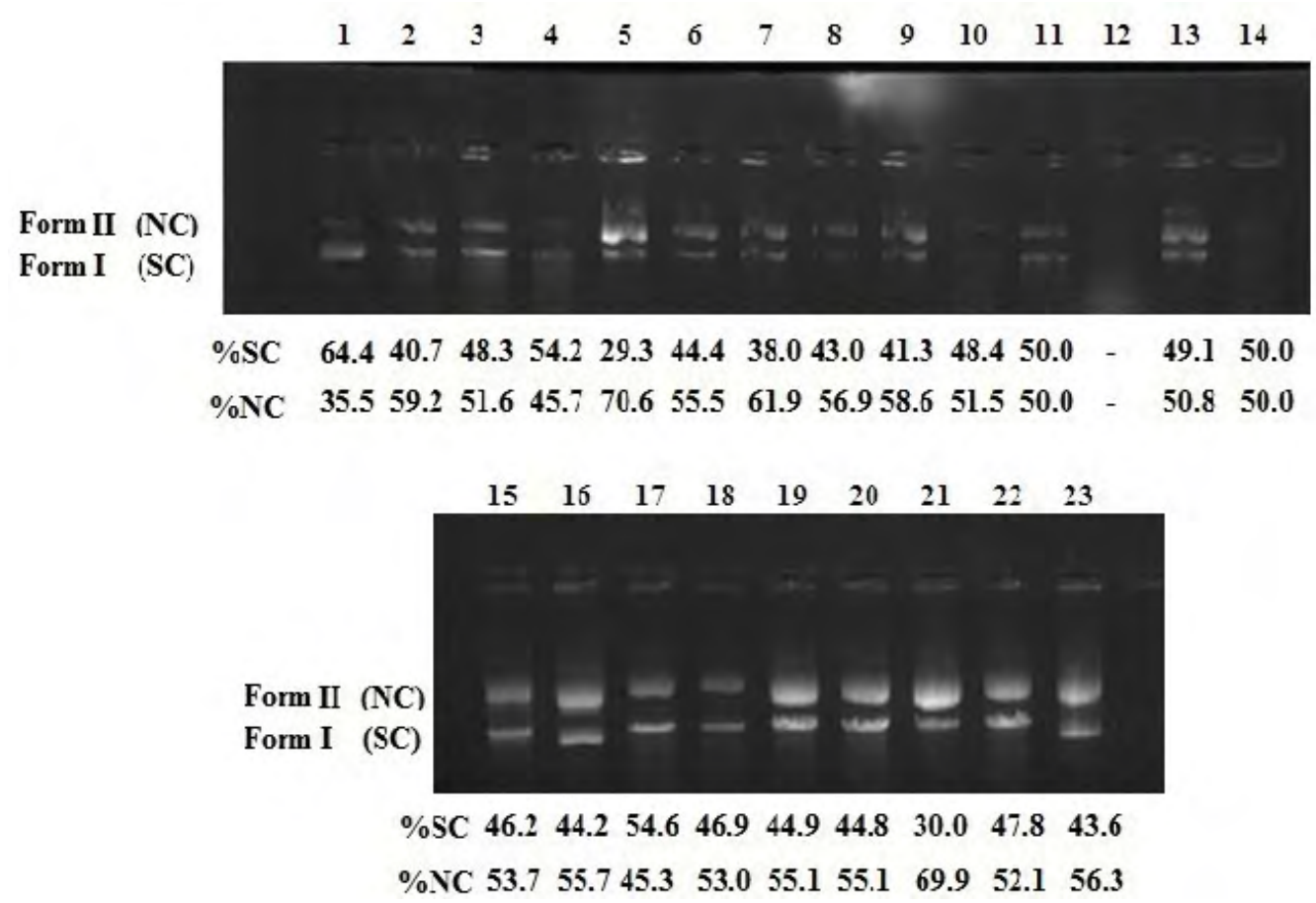

Figure 4: Plasmid DNA photocleavage of oxadiazoles 4a-u. Lane 1: Control plasmid DNA+UV+DMSO, Lane 2: DNA+40 $\mu \mathrm{g}$ 4a, Lane 3: DNA+40 $\mu \mathrm{g}$ 4b, Lane 4: DNA+40 $\mu \mathrm{g} \mathrm{4c,} \mathrm{Lane} \mathrm{5:} \mathrm{DNA+40} \mu \mathrm{g}$ 4d, Lane 6: DNA+40 $\mu \mathrm{g}$ 4e, Lane 7: DNA+40 $\mu \mathrm{g}$ 4f, Lane 8: DNA+40 $\mu \mathrm{g}$ 4g, Lane 9: DNA+40 $\mu \mathrm{g}$ 4h, Lane 10: DNA+40 $\mu \mathrm{g} 4 \mathrm{i}$,

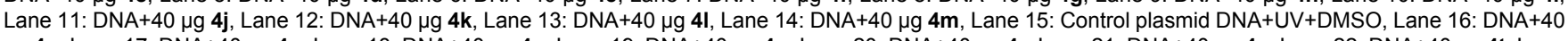

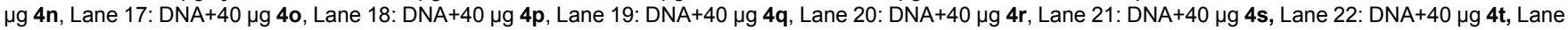
23: DNA+40 $\mu \mathrm{g} 4 \mathrm{u}$, respectively. Values under each lane represent percentage contribution of Form I and II.

\section{Acknowledgements}

The authors are grateful to the Chairman, Maharishi Markandeshwar University, Mullana, Ambala, Haryana for providing the necessary research facilities.

\section{References}

1. Kaur K, Kumar V, Gupta GK, Sharma AK (2014) Isoxazoline containing natural products as anticancer agents: a review. Eur J Med Chem 77: 121-133.

2. LuX, Liu X, Wan B, Franzblau SG, Chen L, et al. (2012) Synthesis and evaluation of anti-tubercular and antibacterial activities of new 4-(2,6-dichlorobenzyloxy) phenyl thiazole, oxazole and imidazole derivatives. Part 2. Eur J Med Chem 49: 164-171.

3. Kumar V, Kaur K, Gupta GK, Sharma AK (2013) Pyrazole containing natural products: synthetic preview and biological significance. Eur J Med Chem 69: 735-753

4. Sharma AK, Naithani R, Kumar V, Sandhu SS (2011) Iron regulation in tuberculosis research: promise and challenges. Curr Med Chem 18: 17231731.

5. Aggarwal R, Kumar V, Tyagi P, Singh SP (2006) Synthesis and antibacterial activity of some new 1-heteroaryl-5-amino-3H/methyl-4-phenylpyrazoles. Bioorg Med Chem 14: 1785-1791.

6. Kumar V, Aggarwal R, Singh SP (2006) The reaction of hydroxylamine with aryl trifluoromethyl- $\beta$-diketones: Synthesis of 5 -hydroxy-5-trifluoromethyl- $\Delta$ 2 -isoxazolines and their dehydration to 5-trifluoromethylisoxazoles. Fluorine Chem 127: 880-888.

7. Kumar V, Aggarwal R, Singh SP, Elguero JJ (2006) The reaction of aryl and heteroarylhydrazines with aryl-trifluoromethyl $\beta$-diketones. Heterocyclic Chem 43: $1003-1014$

8. Kumar V, Aggarwal R, Singh SP (2007) Synthesis of some new 1-(6-fluorobenzothiazol-2-yl)-3-(4-fluro-phenyl)-5-arylpyrazolines and their iodine(III)mediated oxidation to corresponding pyrazoles. Ind JChem 46B: $1332-1336$.
9. Kumar V, Kaur K, Karelia DN, Beniwal V, Gupta GK, et al. (2014) Synthesis and biological evaluation of some 2-(3,5-dimethyl-1 H-pyrazol-1-yl)-1arylethanones: antibacterial, DNA photocleavage, and anticancer activities. Eur J Med Chem 81: 267-276.

10. Kumar V, Aggarwal R, Gupta GK (2013) Synthesis of some new 3,5-diamino4-(4-fluorophenylazo)-1-aryl/heteroarylpyrazoles as antimicrobial agents. Med Chem Res 22: 3566-3573.

11. Mohareb RM, El-Sayed NN, Abdelaziz MA (2012) Uses of cyanoacetylhydrazine in heterocyclic synthesis: novel synthesis of pyrazole derivatives with antitumor activities. Molecules 17: 8449-8463.

12. Ravala JP, Shaha AB, Patela NH (2011) Synthesis and anti-tubercular activity of novel pyrazol-5(H)-one derivatives. Eur J Chem 2: 238-342.

13. Al-Ayed AS (2011) Synthesis of new substituted chromen[4,3-c]pyrazol-4-ones and their antioxidant activities. Molecules 16: 10292-10302.

14. Kumar V, Kaur K, Gupta GK, Gupta AK, Kumar S (2013) Developments in synthesis of the anti-inflammatory drug, celecoxib: a review. Recent Pat Inflamm Allergy Drug Discov 7: 124-134.

15. Mariappan G, Saha BP, Sutharson L, Haldar A (2010) Synthesis and bioactivity evaluation of pyrazolone derivatives. Ind J Chem 49B: 1671-1674.

16. Kumar V, Aggarwal R, Tyagi P, Singh SP (2005) Synthesis and antibacteria activity of some new 1-heteroaryl-5-amino-4-phenyl-3-trifluoromethylpyrazoles. Eur J Med Chem 40: 922-927.

17. Gupta GK, Kumar V, Kumar V (2011) Pyrazoles as potential anti-obesity agents. Res J Chem Environ 15: 90-103.

18. Abdel-Aziz M, Abuo-Rahma GD, Hassan AA (2009) Synthesis of novel pyrazole derivatives and evaluation of their antidepressant and anticonvulsant activities. Eur J Med Chem 44: 3480-3487.

19. Merugu KS, Kurnool A, Lakkakula VK, Pujari JN, Abbavaram BR, et al. (2011) Synthesis, characterization and biological activities of 2,5-disubstituted 1,3,4oxadiazoles. Der PharmaChemica 3: 130-137. 
Citation: Kumar V, Kumar M, Beniwal V, Gupta GK, Kumar S, et al. (2016) Synthesis of Some Aroylhydrazones and 2,5-Disubstituted-1,3,4Oxadiazoles as DNA Photocleaving Agents. Med chem (Los Angeles) 6: 474-485. doi:10.4172/2161-0444.1000386

20. Bakht MA, Yar MS, Abdel-Hamid SG, Qasoumi SI, Samad A (2010) Molecular properties prediction, synthesis and antimicrobial activity of some newer oxadiazole derivatives. Eur J Med Chem 45: 5862-5869.

21. Chandrakantha B, Shetty P, Nambiyar V, Isloor N, Isloor AM (2010) Synthesis, characterization and biological activity of some new ,3,4-oxadiazole bearing 2-flouro-4-methoxy phenyl moiety. Eur J Med Chem 45: 1206-1210.

22. Salahuddin SM, Mazumder A, Abdullah MM (2012) Synthesis, characterization and antimicrobial activity of 1,3,4-oxadiazole bearing $1 \mathrm{H}$-benzimidazole derivatives. Arab J Chem.

23. Dash S, Kumar BA, Singh J, Maiti BC, Maity TK (2011) Synthesis of some novel 3,5-disubstituted 1,3,4-oxadiazole derivatives and anticancer activity on EAC animal model. Med Chem Res 20: 1206-1213.

24. Zhang ZM, Zhang XW, Zhao ZZ, Yan R, Xu R, et al. (2012) Synthesis, biological evaluation and molecular docking studies of ,3,4-oxadiazole derivatives as potential immunosuppressive agents. Bioorg Med Chem 20: 3359-3367.

25. Tang JF, Lv XH, Wanga XL, Sun J, Zhang YB, et al. (2012) Design, synthesis, biological evaluation and molecular modeling of novel 1,3,4-oxadiazole derivatives based on Vanillic acid as potential immunosuppressive agents. Bioorg Med Chem 20: 4226-4236.

26. Bajaj S, Asati V, Singh J, Roy PP (2015) 1,3,4-Oxadiazoles: An emerging scaffold to target growth factors, enzymes and kinases as anticancer agents. Eur J Med Chem 97: 124-141.

27. Gurupadaswamy HD, Girish V, Kavitha CV, Raghavan SC, Khanum SA (2013) Synthesis and evaluation of 2,5-di(4-aryloylaryloxymethyl)-,3,4-oxadiazoles as anti-cancer agents. Eur J Med Chem 63: 536-543.

28. Kumar D, Sundaree S, Johnson EO, Shah K (2009) An efficient synthesis and biological study of novel indolyl-,3,4-oxadiazoles as potent anticancer agents. Bioorg Med Chem Lett 19: 4492-4494.

29. Kulkarni AKD, Patil SA, Naik VH, Badam PS (2010) DNA cleavage and antimicrobial investigation of $\mathrm{Co}(\mathrm{II}), \mathrm{Ni}(\mathrm{II})$, and $\mathrm{Cu}(\mathrm{II})$ complexes with triazole Schiff bases: synthesis and spectral characterization. MedChem Res 20: 346354.

30. Taj T, Kamble RR, Kattimani PP, Badami BV (2012) Synthetic utility of sydnones: synthesis of pyrazolinesderivatized with 1,2,4-triazoles as antihyperglymic, antioxidant agents and their DNA cleavage study. Med Chem Res 2: 37093719.

31. Hanumanagoud $\mathrm{H}$, Basavaraja KMJ (2012) Synthesis, antibacterial, antifungal activity and DNA cleavage study of 3-(7-methoxy-benzofuran-2-yl)-5-aryl$4 \mathrm{H}-[1,2,4]$ triazoles. Chem Pharm Res 4: 5165-5171.

32. Raman N, Raja SJ (2007) DNA cleavage, structural elucidation and antimicrobial studies of three novel mixed ligand Schiff base complexes of coppe (II). J Serb Chem Soc 72: 983-992.

33. Kurdekar GS, Puttanagouda SM, Kulkarni NV, Budagumpi S, Revankar VK (2012) Synthesis, characterization, antibiogram and DNA binding studies of novel $\mathrm{Co}(\mathrm{II}), \mathrm{Ni}(\mathrm{II}), \mathrm{Cu}(\mathrm{II})$, and $\mathrm{Zn}(\mathrm{II})$ complexes of Schiff base ligands with quinoline core. MedChem Res 20: 421-429.
34. Manjunatha K, Poojary B, Lobo PL, Fernandes J, Kumari NS (2010) Synthesis and biological evaluation of some ,3,4-oxadiazole derivatives. Eur J Med Chem 45: 5225-5233.

35. Rajput AP, Rajput SS (2013) A Novel Method For The Synthesis Of FormylPyrazoles Using Vilsmeier-Haack Reaction. Int J Pharm Pharm Sci 3: 346-351.

36. Kumar M, Kumar V, Gupta GK (2014) synthesis antibacterial evaluation and SAR study of some novel 3-aryl/heteroaryl-9-methyl-1,2,4-triazolo-[4,3-a]quinoline derivatives. Med Chem Res 24: 1857-1868.

37. Tegginamath G, Kamble RR, Kattimani PP, Margankop SB (2011) Synthesis of 3-aryl-4-(\{2-[4-(6-substituted-coumarin-3-yl)-I,3-thiazol-2-yl]-hydrazinylidene\}methyl/ethyl)-sydnones using silica sulfuric acid and their antidiabetic, DNA cleavage activity. Arab J Chem.

38. Jha KK, Samad A, Kumar Y, Shaharyar M, Khosa RL, et al. (2010) Design, synthesis and biological evaluation of ,3,4-oxadiazole derivatives. Eur $\mathrm{J}$ Med Chem 45: 4963-4967.

39. Rostamizadeh S, Ghamkhar SA (2008) A mild and facile method for one pot synthesis of 2,5-di-substituted 1,3,4-oxadiazoles at room temperature. Chin Chem Lett 19: 639-642.

40. de Oliveira CS, Lira BF, dos Santos Falcão-Silva V, Siqueira JP Jr, BarbosaFilho JM, et al. (2012) Synthesis, molecular properties prediction, and anti-staphylococcal activity of $\mathrm{N}$-acylhydrazones and new ,3,4-oxadiazole derivatives. Molecules 17: 5095-5107.

41. Aggarwal R, Kumar R, Kumar VJ (2007) A facile and rapid one-pot synthesis of 1, 4-diaryl-2-mercaptoimidazoles under solvent-free conditions. Sulfur Chem 28: $617-623$

42. Vorvoglis A (1997) Chemical transformations induced by hypervalent iodine reagents. Tetrahedron 53: 1179-1255.

43. Zhdankin VV (2009) Hypervalent iodine (III) reagents in organic synthesis Arkivoc 1: 1-62.

44. Kumar $P$ (2012) An environmentally benign and solvent-free synthesis of 3 -aryl[1,2,4]triazolo[4,3-a]pyridines and 1-aryl-5-methyl[1,2,4]triazolo[4,3-a] quinolines using phenyliodine bis(trifluoroacetate) or iodobenzene diacetate. Chem Heterocycl Compds 47: 1237-1243.

45. Prakash O, Hussain K, Aneja DK, Sharma C, Aneja KR (2011) facile iodine(III)mediated synthesis of 3-(3-aryl-1-phenyl-1H-pyrazol-4-yl)-[1,2,4]triazolo[4,3-a] pyridines via oxidation of 2-((3-aryl-1-phenyl-1H-pyrazol-4-yl)methylene)-1(pyridin-2-yl)hydrazines and their antimicrobial evaluations. Org Med Chem Lett 1: 1.

46. Prakash O, Kumar M, Kumar R, Sharma C, Aneja KR (2010) Hypervalen iodine(III) mediated synthesis of novel unsymmetrical 2,5-disubstituted ,3,4-oxadiazoles as antibacterial and antifungal agents. Eur J Med Chem 45: 4252-4257.

47. Yang R, Dai LJ (1993) Hypervalent iodine oxidation of $\mathrm{N}$-acylhydrazones and $\mathrm{N}$-phenylsemicarbazone: an efficient method for the synthesis of derivatives of 1,3,4-oxadiazoles and DELTA 3-1,3,4-oxadiazolines. Org Chem 58: 3381 3383. 